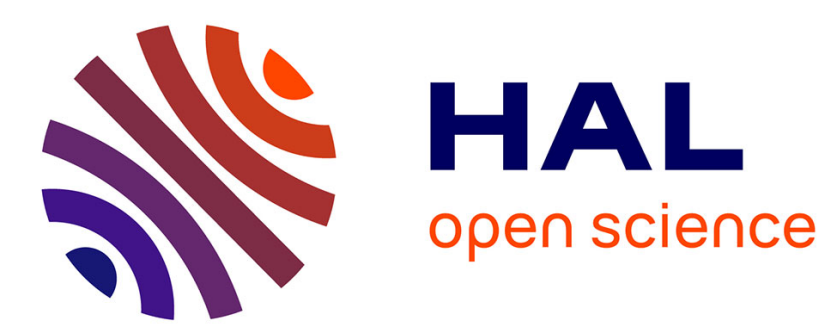

\title{
Simplicity-based recovery of finite-alphabet signals for large-scale MIMO systems
}

\author{
Zahran Hajji, Abdeldjalil Aissa El Bey, Karine Amis Cavalec
}

\section{To cite this version:}

Zahran Hajji, Abdeldjalil Aissa El Bey, Karine Amis Cavalec. Simplicity-based recovery of finitealphabet signals for large-scale MIMO systems. Digital Signal Processing, 2018, 80, pp.70-82. 10.1016/j.dsp.2018.05.012 . hal-01811519

\section{HAL Id: hal-01811519 \\ https://hal.science/hal-01811519}

Submitted on 9 Jun 2018

HAL is a multi-disciplinary open access archive for the deposit and dissemination of scientific research documents, whether they are published or not. The documents may come from teaching and research institutions in France or abroad, or from public or private research centers.
L'archive ouverte pluridisciplinaire HAL, est destinée au dépôt et à la diffusion de documents scientifiques de niveau recherche, publiés ou non, émanant des établissements d'enseignement et de recherche français ou étrangers, des laboratoires publics ou privés. 


\title{
Simplicity-based recovery of finite-alphabet signals for large-scale MIMO systems
}

\author{
Zahran Hajji ${ }^{1}$, Abdeldjalil Aïssa-El-Bey ${ }^{1}$, Karine Amis ${ }^{1}$ \\ IMT Atlantique, UMR CNRS 6285 Lab-STICC, UBL, F-29238 Brest, France
}

\begin{abstract}
In this paper, we consider the problem of finite-alphabet source separation in both determined and underdetermined large-scale systems. First, we address the noiseless case and we propose a linear criterion based on $\ell_{1}$-minimization combined with box constraints. We investigate also the system conditions that ensure successful recovery. Next, we apply the approach to the noisy massive MIMO transmission and we propose a quadratic criterion-based detector. Simulation results show the efficiency of the proposed detection methods for various QAM modulations and MIMO configurations. We mention that there is no change in the computational complexity when the constellation size increases. Moreover, the proposed method outperforms the classical Minimum Mean Square Error (MMSE)-based detection algorithms.

Keywords: Compressed sensing, source separation, underdetermined system, sparsity, simplicity, massive MIMO.
\end{abstract}

\section{Introduction}

Source separation problems in digital signal processing deal with the recovery of original source signals from the observed mixture signal $[1,2]$. 
In the overdetermined case, the number of observations exceeds the number of sources and the recovery is possible without making strong assumptions about the sources or the mixing parameters [3]. However, the separation problem becomes more difficult if the number of underlying sources is larger than the number of observations. Separation of such underdetermined mixtures requires the separation algorithm to exploit additional information about the source signals and the mixing parameters compared to the overdetermined case. Compressed sensing (CS) technique $[4,5]$ has attracted considerable attention as it promises to surpass the traditional limits of sampling theory [6]. It is a signal processing technique to efficiently acquire and reconstruct signals, by finding solutions to underdetermined linear systems. It exploits the sparsity of the signal to recover it and thus, it uses far fewer samples than required by the sampling theorem [7, 8]. A source is sparse in a given representation domain if most of its elements are close to zero. The CS technique requires the sparsity of the sources which restricts its application [9]. In [10], the authors proposed to apply the CS technique to solve underdetermined real-valued finite-alphabet source recovery problems. They introduced a suitable sparse decomposition to derive a sparse recovery problem solvable by CS techniques. In [11], Mangasarian et al. treated the binary alphabet case and showed that the source can be successfully recovered by resolving a linear program with higher detection probability as the number of observations exceeds half the number of sources. Mangasarian analysis remains true for all size-2 alphabets [10]. The recovery problem is especially important in data communications which is the field of interest of this paper and in image processing. 
In this paper, we address the problem of finite-alphabet signal recovery for large-scale MIMO systems involving high-dimensional problems. We first consider the noiseless general case as in $[10,11]$. However, contrary to the aforementioned references, we study the case of complex-valued alphabets with any cardinality and complex-valued mixing matrices. We then propose a linear program for CS technique based on signal simplicity. Simplicity was first introduced by Donoho et al. in [12]. A signal is considered simple if most of its elements are equal to the extremes of the finite alphabet. We then prove that this proposed scheme provides the same efficient recovery performance as the schemes in $[10,13,14]$ with lower computational complexity especially when the alphabet cardinality is high. We also show that the recovery scheme performs better when the dimensions of the mixing matrix increase.

In a second step, we propose to apply the principle to noisy massive MIMO transmission, which can be considered as a particular case of largescale MIMO systems. Massive MIMO technology has been selected in the $5 \mathrm{G}$ standard definition as a solution to provide higher throughput under spectrum limitations [15]. It promises significant gains and offers the ability to serve more users at higher data rates with better reliability. Large number of antennas and/or users is involved, which makes receiver design critical from complexity point of view.

Research for high-performance receiver design that can lead to practical realization of massive MIMO systems is both nascent as well as promising. Sphere decoders [16], which are based on maximum likelihood (ML), require an exhaustive search within an hypersphere whose dimensions remain high in the massive MIMO case, yielding computationally-unsolvable detection. 
Usual linear equalizers such as minimum mean square error (MMSE) [17, 18] and zero-forcing (ZF) [19] have lower computational complexity but degraded error rate performance compared to ML detectors, especially in the undetermined uncoded case. Successive interference cancellation (SIC) schemes were proposed such as MMSE-SIC in [20] to enhance the linear equalizer performance at the expense of higher complexity. Further error rate decrease was achieved by combining SIC and lattice reduction (LR) schemes as done for instance in the MMSE-SIC-LR studied in [21]. In this paper, we address the problem of detection in both determined and underdetermined systems. Underdetermined configuration is expected in future 5G system uplink, as the number of connected users times their transmit antenna number could be much higher than the base station receive antenna number. To carry out this study, we first extend the noiseless detection algorithm to the noisy case to design a low-complexity detector which exploits the simplicity of sources and we show its efficiency compared to existing detection techniques by investigating the error rate performance and the computational complexity.

In this paper, we deal with the problem of recovery of finite-alphabet signals in both determined and underdetermined large-scale systems by exploiting the simplicity property of the alphabet. Compared to previous work $[10,11,13,14]$, the proposed criterion applies whatever the alphabet size or domain (real or complex-valued) and achieves the best performance with computational cost independent of the alphabet size. The efficiency of the proposed simplicity-based technique can be explained by the added constraints. These constraints ensure that some estimated output are highly reliable and will not contribute to the error propagation. Thus, the error 
propagation is reduced compared to iterative MMSE-based techniques.

Our contributions are: $(i)$ a new criterion based on the simplicity property of finite-alphabet signals $(\boldsymbol{i} i)$ the necessary condition of successful recovery in the noise-free case (iii) the extension of the proposed criterion to the noisy case $(\boldsymbol{i v})$ the theoretical probability density function of the proposed algorithm output $(\boldsymbol{v})$ the theoretical symbol error probability in the case of M-QAM modulations.

This paper is organized as follows. Section 2 describes the system models considered in the following and provides an overview of state-of-the-art compressed sensing techniques. Section 3 deals with source separation problem in noise-free systems. Section 4 describes how the proposed source separation scheme is extended to be applied in massive MIMO systems. Finally, Section 5 concludes the paper.

Notations: Boldface upper case letters and boldface lower case letters denote matrices and vectors, respectively. For transpose, transpose conjugate and conjugate operations we use $(.)^{T},(.)^{H}$ and $(.)^{*}$, respectively. $\otimes$ is the Kronecker product. $\boldsymbol{I}_{k}$ is the $k \times k$ identity matrix and $\mathbf{1}_{k}$ is the all-one size- $k$ vector. Let $\boldsymbol{z} \in \mathbb{C}^{k}$ be a complex-valued vector of size $k$. We denote by $\underline{\boldsymbol{z}} \in \mathbb{R}^{2 k}$ its real-valued transformed vector which can be defined by $\underline{\boldsymbol{z}}=$ $(\operatorname{Re}(\boldsymbol{z}) \operatorname{Im}(\boldsymbol{z}))^{T}$. Let also $\boldsymbol{H} \in \mathbb{C}^{n \times N}$ a complex-valued matrix with size $n \times N$, we denote by $\underline{\boldsymbol{H}} \in \mathbb{R}^{2 n \times 2 N}$ its real-valued matrix version, which is defined by $\underline{\boldsymbol{H}}=\left(\begin{array}{cc}\operatorname{Re}(\boldsymbol{H}) & -\operatorname{Im}(\boldsymbol{H}) \\ \operatorname{Im}(\boldsymbol{H}) & \operatorname{Re}(\boldsymbol{H})\end{array}\right)$. erfc $(\cdot)$ is the complementary error function. It is defined as $\operatorname{erfc}(x)=\frac{2}{\sqrt{\pi}} \int_{x}^{\infty} e^{-t^{2}} d t . \quad \delta(\cdot)$ is the Dirac delta function and $\mathbb{1}_{\mathcal{A}}(\cdot)$ is the indicator function of the subset $\mathcal{A}$. 


\section{System model and overview}

\subsection{Noise-free large-scale systems}

We first consider the noise-free mixing model, which can be described by the following linear system:

$$
\boldsymbol{y}=\boldsymbol{H} \boldsymbol{x},
$$

where $\boldsymbol{x} \in \mathbb{C}^{N}$ is the $N \times 1$ complex-valued source vector, $\boldsymbol{y} \in \mathbb{C}^{n}$ is the $n \times 1$ complex-valued observation vector and $\boldsymbol{H} \in \mathbb{C}^{n \times N}$ is an $n \times N$ complexvalued random matrix. We assume that the components of $\boldsymbol{H}$ are independent and circularly symmetric Gaussian with zero mean and unit variance. The vector $\boldsymbol{x}$ belongs to a complex-valued finite alphabet. It can be decomposed from its real and imaginary parts as $\boldsymbol{x}=\boldsymbol{a}+j \boldsymbol{b}$ where $(\boldsymbol{a}, \boldsymbol{b}) \in \mathcal{F}^{N} \times \mathcal{F}^{N}$ and $\mathcal{F}=\left\{\alpha_{1}, \alpha_{2}, . ., \alpha_{p}\right\}$. The equivalent real-valued linear system can then be written as:

$$
\underline{\boldsymbol{y}}=\underline{\boldsymbol{H} \boldsymbol{x}}, \quad \underline{\boldsymbol{x}} \in \mathcal{F}^{2 N} .
$$

We assume that the elements of $\mathcal{F}$ are equiprobable under the realization of $\underline{\boldsymbol{x}}$. Then, our problem is the recovery of $\underline{\boldsymbol{x}}$ from $\underline{\boldsymbol{y}}$ given $\underline{\boldsymbol{H}}$ and $\mathcal{F}$.

A special case was introduced by Mangasarian et al. in [11]. They considered the real-valued problem with $\boldsymbol{H}$ an $n \times N$ real-valued generic random matrix $^{1}$ and the vector $\boldsymbol{x}$ belonging to the real-valued finite alphabet $\{-1,1\}$. In this case, $\boldsymbol{x}$ can be recovered by solving the $\ell_{\infty}$-norm minimization

$$
\left(P_{\infty}\right): \underset{x}{\arg \min }\|\boldsymbol{x}\|_{\infty} \quad \text { subject to } \quad \boldsymbol{y}=\boldsymbol{H} \boldsymbol{x} .
$$

${ }^{1}$ A matrix $\boldsymbol{H}$ is a generic random matrix if all sets of $\ell$ columns are linearly independent with probability 1 and each column is symmetrically distributed about the origin.[11] 
This optimization system was reformulated by a linear programming problem and the authors proved that the probability of successful recovery equals the probability that all of the columns of the generic random matrix lie in the same hemisphere. This probability is determined by the following theorem.

Theorem 2.1. Wendel [22] Let $\boldsymbol{H}$ an $n \times N$ real-valued generic random matrix. The probability that all of its columns lie in the same hemisphere is precisely equal to

$$
P_{n, N}=2^{-N+1} \sum_{i=0}^{n-1}\left(\begin{array}{c}
N-1 \\
i
\end{array}\right) .
$$

As an extension of this work, the authors in [10] generalized the problem to all size-2 constellations $\left[\alpha_{1}, \alpha_{2}\right]$ thanks to a simple translation.

In the complex case given by Eq. (1), we demonstrate in the Appendix A that given the properties of the complex-valued matrix $\boldsymbol{H}$, its real-valued matrix version $\underline{\boldsymbol{H}}$ is random generic. Then, the probability of successful recovery is equal to the probability that all of the columns of $\boldsymbol{H}$ lie in the first quadrant of the complex plane, that is to say the probability that all of the columns of $\underline{\boldsymbol{H}}$ lie in the same hemisphere. According to Wendel's theorem, this probability denoted by $Q_{n, N}$ equals $P_{2 n, 2 N}$ :

$$
Q_{n, N}=P_{2 n, 2 N}=2^{-2 N+1} \sum_{i=0}^{2 n-1}\left(\begin{array}{c}
2 N-1 \\
i
\end{array}\right) .
$$

In the context of underdetermined systems where the number of observations is less than the number of sources, the CS is a good candidate to separate the sources, provided the source vector is sparse. In the case of interest, the source vector isn't sparse and the symbols belong to a finite constellation with non-null elements. In order to apply recovery techniques similar to 
the Basis Pursuit (BP), the authors proposed in [13] a solution based on a suitable sparse transform to benefit from the combination of sparsity and finite-alphabet constraints. They succeeded in decomposing any element of the set $\mathcal{F}^{2 N}$ as a sparse vector in $\mathbb{R}^{2 N p}$. The sparse vector is composed of $2 N$ consecutive $p$-uples, such that each $p$-uple contains one 1 and $p-1$ zeros. By proceeding so, the problem of detection becomes equivalent to a problem of sparse recovery from incomplete measurements. This problem can be seen as minimization of the $\ell_{0}$-norm of the sparse-transformed vector subject to two constraints. The first is $\underline{\boldsymbol{y}}=\underline{\boldsymbol{H}} \boldsymbol{B}_{f} \boldsymbol{s}$ where $\boldsymbol{s}$ is the sparsetransform of $\boldsymbol{x}$ and $\boldsymbol{B}_{f}=\boldsymbol{I}_{2 N} \otimes \boldsymbol{f}^{T}$ is the transformation matrix which is defined as the Kronecker product of the identity matrix and the realvalued alphabet vector $\boldsymbol{f}=\left[\alpha_{1}, \alpha_{2}, . . \alpha_{p}\right]^{T}$. The second is the uniqueness constraint which reads $\boldsymbol{B}_{1} \boldsymbol{s}=\mathbf{1}_{N}$ where $\boldsymbol{B}_{1}=\boldsymbol{I}_{2 N} \otimes \mathbf{1}_{p}^{T}$. It imposes the sparse reconstruction of the searched vector. However, an $\ell_{0}$-minimization problem is NP-hard. Therefore, to exploit the sparsity to solve the recovery and to have a problem with feasible complexity, the $\ell_{0}$-minimization is relaxed to an $\ell_{1}$-minimization, by mimicking literature on sparse reconstruction [23]. The optimization problem now reads

$$
\left(P_{S A, 1}\right): \underset{s}{\arg \min }\|\boldsymbol{s}\|_{1} \quad \text { subject to } \quad \underline{\boldsymbol{y}}=\underline{\boldsymbol{H}} \boldsymbol{B}_{f} \boldsymbol{s}, \quad \boldsymbol{B}_{1} \boldsymbol{s}=\mathbf{1}_{2 N},
$$

where $s$ is the resulted sparse vector which contains $2 N p$-tuples, each with a single element different from zero.

The main drawback of $\left(P_{S A, 1}\right)$ is its complexity which highly depends on the alphabet size. This makes it less interesting for higher sizes. To address the complexity issue, the authors proposed another sparse decomposition which is done in two steps [24]. The first is a binary decomposition as proposed 
in [25] which transforms the elements of a vector in $\mathcal{F}$ into a size- $4 N \log _{2}(p)$ vector of binary elements $\{-1,1\}$. The second step is the application of the previous sparse decomposition to the resulting binary vector. The problem becomes the recovery of a half-sparse vector with half of null elements. The resulting problem, denoted by $\left(P_{H S A, 1}\right)$, reads

$$
\left(P_{H S A, 1}\right): \arg \min \|\boldsymbol{s}\|_{1} \quad \text { subject to } \quad \underline{\boldsymbol{y}}=\underline{\boldsymbol{H}} \boldsymbol{B}_{\beta} \boldsymbol{B}_{p} \boldsymbol{s}, \quad \boldsymbol{B}_{1} \boldsymbol{s}=\mathbf{1}_{2 \ell N}, \quad(7)
$$

where $\ell=\log _{2}(M)=2 k, \boldsymbol{B}_{1}=\boldsymbol{I}_{\ell N} \otimes \mathbf{1}_{2}^{T}, \boldsymbol{B}_{\beta}=\boldsymbol{I}_{N} \otimes \boldsymbol{\beta}$ with $\boldsymbol{\beta}=$ $\left[2^{k-1}, \ldots, 2^{1}, 2^{0}\right]$ and $\boldsymbol{B}_{\rho}=\boldsymbol{I}_{\ell N} \otimes \boldsymbol{\rho}$ with $\boldsymbol{\rho}=[-1,1] . \quad \boldsymbol{B}_{\beta}$ defines the binary decomposition and $\boldsymbol{B}_{\rho}$ the half-sparse decomposition.

$\left(P_{H S A, 1}\right)$ is less complex than $\left(P_{S A, 1}\right)$ while achieving the same successful recovery probability. It reduces by about $\left(\frac{2 \log _{2}(p)}{p}\right)^{2}$ the computation cost $[24]$.

\subsection{Noisy massive MIMO systems}

Let us study the case of noisy massive MIMO transmission. We consider a $K$-user system over a flat fading channel, where each user has $N_{t}$ transmit antennas and let $N=K \times N_{t}$. No user cooperation for transmission nor precoding scheme is taken into account. At the base station side, we assume $n$ equivalent receive antennas (base station cooperation through the base station controller is possible). We assume a perfect channel state information (CSI) at the base station. In this paper, we focus on the uplink of the communication system. The received signal reads

$$
\boldsymbol{y}=\boldsymbol{H} \boldsymbol{x}+\boldsymbol{\zeta}
$$

where $\boldsymbol{H}$ is an $n \times N$ random channel matrix, $\boldsymbol{x}$ is the $N \times 1$ data vector, and $\zeta$ is the $n \times 1$ complex circularly symmetric additive Gaussian noise vector 
with zero mean and covariance matrix equal to $2 \sigma^{2} \boldsymbol{I}_{n}$. We assume that the components of $\boldsymbol{x}$ belong to an $M$-QAM modulation alphabet such that $M=p^{2}$ (square QAM constellation). The modulation alphabet, denoted by $\mathcal{Q}$, is defined by $\mathcal{Q}=\left\{q_{1}, q_{2}, \ldots, q_{M}\right\}$. We associate to $\mathcal{Q}$ the symbol vector $\boldsymbol{q}=\left[q_{1}, q_{2}, \ldots, q_{M}\right]$ where $q_{i}=a_{i}+j b_{i}, i \in\{1 . . M\}$ with $\left(a_{i}, b_{i}\right) \in \mathcal{F} \times \mathcal{F}$. Then, the system model is equivalent to the following real-valued one:

$$
\underline{\boldsymbol{y}}=\underline{\boldsymbol{H} \boldsymbol{x}}+\underline{\boldsymbol{\zeta}}, \quad \underline{\boldsymbol{x}} \in \mathcal{F}^{2 N} .
$$

The main objective is to estimate $\underline{\boldsymbol{x}}$ from $\underline{\boldsymbol{y}}$ given $\underline{\boldsymbol{H}}$ and $\mathcal{F}$ by exploiting the sparse decomposition of $\underline{\boldsymbol{x}}$. This objective can be achieved by an $\ell_{1}$ minimization problem that involves $\epsilon$ as a variable parameter depending on the current signal-to-noise ratio (SNR) value to ensure that the estimated vector is close to the emitted one.

The authors proposed in [14] to apply the sparse decomposition and solve the noisy MIMO recovery problem by the following constrained $\ell_{1}$ minimization:

$$
\underset{s}{\arg \min }\|\boldsymbol{s}\|_{1} \quad \text { subject to } \quad\left\|\underline{\boldsymbol{y}}-\underline{\boldsymbol{H}} \boldsymbol{B}_{f} \boldsymbol{s}\right\|_{2} \leq \epsilon, \quad \boldsymbol{B}_{1} \boldsymbol{s}=\mathbf{1}_{2 N} .
$$

The efficiency of the algorithm depends on the choice of $\epsilon$. To counterbalance the critical choice of the parameter, they proposed another quadratic optimization system which can be seen as relaxation of the (ML) in another quadratic system with $\ell_{0}$-equality as a constraint to ensure the sparsity of the searched vector. The $\ell_{1}$ constraint is equivalent to a positivity constraint. The result is a quadratic programming model with linear equality constraints and non-negative variables. It can be resolved by polynomial-complexity al- 
gorithms. In the end, the optimization problem reads

$$
\left(P_{S A, 2}\right): \underset{s}{\arg \min }\left\|\underline{\boldsymbol{y}}-\underline{\boldsymbol{H}} \boldsymbol{B}_{f} \boldsymbol{s}\right\|_{2} \quad \text { subject to } \quad \boldsymbol{B}_{1} \boldsymbol{s}=\mathbf{1}_{2 N}, \quad \boldsymbol{s} \geq 0 .
$$

Like $\left(P_{S A, 1}\right)$, the complexity of $\left(P_{S A, 2}\right)$ highly depends on the constellation size. The same decomposition as used in $\left(P_{H S A, 1}\right)$ can be applied to obtain the reduced-complexity problem $\left(P_{H S A, 2}\right)$ which reads [24]

$$
\left(P_{H S A, 2}\right): \underset{s}{\arg \min }\left\|\underline{\boldsymbol{y}}-\underline{\boldsymbol{H}} \boldsymbol{B}_{\beta} \boldsymbol{B}_{\rho} \boldsymbol{s}\right\|_{2} \quad \text { subject to } \quad \boldsymbol{B}_{1} \boldsymbol{s}=\mathbf{1}_{2 l N}, \quad \boldsymbol{s} \geq 0 .
$$

In this paper, we present a new method for compressive sensing that does not require the sparsity of the signal to be recovered. It exploits the alphabet properties and looks for a solution in a convex space containing the alphabet elements. Compared to previously described methods $\left(P_{H S A, i}\right)$ and $\left(P_{S A, i}\right)$, it brings further complexity reduction to adapt to high finite-alphabet size with recovery performance conservation.

\section{Simplicity property exploitation to solve the noise-free recovery}

\subsection{Proposed method definition and theoretical study}

In this section, we consider the noise-free system $\underline{\boldsymbol{y}}=\underline{\boldsymbol{H} \boldsymbol{x}}, \underline{\boldsymbol{x}} \in \mathcal{F}^{2 N}$ and we propose a recovery scheme. The maximum likelihood (ML) detector requires an exhaustive search over all possible mixed symbol vectors and selects the solution that corresponds to the closest point to the searched signal in the known alphabet [26]. In other words, it selects the vector with elements in the alphabet that satisfies the equality $\underline{\boldsymbol{y}}=\underline{\boldsymbol{H} \boldsymbol{x}}$.

$$
\left(P_{M L, 1}\right): \underset{\underline{\boldsymbol{x}}}{\arg \min } \mathbf{1}_{2 N}^{T} \underline{\boldsymbol{x}} \quad \text { subject to } \quad \underline{\boldsymbol{y}}=\underline{\boldsymbol{H} \boldsymbol{x}}, \quad \underline{\boldsymbol{x}} \in \mathcal{F}^{2 N} .
$$


The main drawbacks of this detector are twofold: first, the $\left(P_{M L, 1}\right)$ criterion is not convex and second, it suffers from high computational complexity caused by the exhaustive search over the set $\mathcal{F}^{2 N}$. Herein, we propose a new detection scheme which is based on a relaxation of the ML detector constraint. We relax the solution space $\left\{\underline{\boldsymbol{x}} \in \mathcal{F}^{2 N} \mid \underline{\boldsymbol{y}}=\underline{\boldsymbol{H} \boldsymbol{x}}\right\}$ by substituting it with the convex set $\left\{\underline{\boldsymbol{x}} \in\left[\alpha_{1}, \alpha_{p}\right]^{2 N} \mid \underline{\boldsymbol{y}}=\underline{\boldsymbol{H} \boldsymbol{x}}\right\}$. Exploiting this relaxation, the new resulting optimization problem can be resolved by polynomial algorithms for convex optimization using the following proposition.

Proposition 3.1. $\underline{\boldsymbol{x}} \in\left[\alpha_{1}, \alpha_{p}\right]^{2 N}$ is the unique solution to the problem $\underset{\underline{\boldsymbol{x}}}{\arg \min } \mathbf{1}_{2 N}^{T} \underline{\boldsymbol{x}} \quad$ subject to $\quad \underline{\boldsymbol{y}}=\underline{\boldsymbol{H} \boldsymbol{x}}, \quad \underline{\boldsymbol{x}} \in \mathcal{F}^{2 N}$

if and only if its corresponding vector $\boldsymbol{r} \in \mathbb{R}^{4 N}$ is the unique solution to the optimization problem:

$\underset{\boldsymbol{r}}{\arg \min } \mathbf{1}_{4 N}^{T} \boldsymbol{r} \quad$ subject to $\quad \underline{\boldsymbol{y}}=\underline{\boldsymbol{H}} \boldsymbol{B}_{\alpha} \boldsymbol{r}, \quad \boldsymbol{B}_{1} \boldsymbol{r}=\mathbf{1}_{2 N}, \quad \boldsymbol{r} \geq 0$,

where $\boldsymbol{B}_{\alpha}$ is defined as $\boldsymbol{B}_{\alpha}=\boldsymbol{I}_{2 N} \otimes\left[\alpha_{1}, \alpha_{p}\right]$.

Proof of Proposition 3.1. Let $\mathcal{G}=\left\{\underline{\boldsymbol{x}} \in \mathbb{R}^{2 N} \mid \underline{\boldsymbol{y}}=\underline{\boldsymbol{H} \boldsymbol{x}}, \underline{\boldsymbol{x}} \in\left[\alpha_{1}, \alpha_{p}\right]^{2 N}\right\}$ and $\mathcal{H}=\left\{\underline{\boldsymbol{x}} \in \mathbb{R}^{2 N} \mid \underline{\boldsymbol{y}}=\underline{\boldsymbol{H} \boldsymbol{x}}, \underline{\boldsymbol{x}}=\boldsymbol{B}_{\alpha} \boldsymbol{r} ; \boldsymbol{r} \in \mathbb{R}^{4 N}, \boldsymbol{B}_{1} \boldsymbol{r}=\mathbf{1}_{2 N}\right.$ and $\left.\boldsymbol{r} \geq 0\right\}$. $\mathcal{H}$ stands for the feasible set of the problem defined in (14). Then, it suffices to show the equality between $\mathcal{G}$ and $\mathcal{H}$.

Suppose that $\underline{\boldsymbol{x}} \in \mathcal{G}$. Then the $i$-th element of $\underline{\boldsymbol{x}}$ can be written as $\underline{x}_{i}=$ $r_{2 i} \alpha_{1}+r_{2 i+1} \alpha_{p}$ where $r_{2 i}+r_{2 i+1}=1,0 \leq r_{2 i}, r_{2 i+1} \leq 1$. Thus $\underline{\boldsymbol{x}} \in \mathcal{H}$. Reciprocally $\mathcal{H} \subset \mathcal{G}$. We deduce that $\mathcal{G}=\mathcal{H}$.

It is important to mention that due to the positivity constraint and the fact that the $\ell_{1}$-norm of a vector is the sum of the absolute values of its 
elements, the proposed optimization system is equivalent to the following $\ell_{1}$-minimization problem:

$$
\left(P_{S I, 1}\right): \underset{r}{\arg \min }\|\boldsymbol{r}\|_{1} \text { subject to } \underline{\boldsymbol{y}}=\underline{\boldsymbol{H}} \boldsymbol{B}_{\alpha} \boldsymbol{r}, \quad \boldsymbol{B}_{1} \boldsymbol{r}=\mathbf{1}_{2 N} .
$$

The new optimization problem $\left(P_{S I, 1}\right)$ is a linear programming model with linear equality constraints. It can be solved by the simplex [27] or the interior point methods [28]. In this paper, we take an interest in the algorithms based on the interior point methods. These algorithms start by finding an interior point of the polytope defined by the constraints and then proceed to the optimal solution by moving inside the polytope.

In order to study the necessary and sufficient conditions of the solution uniqueness of the proposed optimization problem, we exploit the geometry of the system model and we utilize a face counting technique [12]. The following theorem gives the solution uniqueness probability from which we can derive the conditions of successful recovery.

Theorem 3.1. (i) Given the alphabet size $p \geq 2$, if $\underline{\boldsymbol{H}}$ is an $2 n \times 2 N$ generic random complex matrix, the probability that $\left(P_{S I, 1}\right)$ has a unique solution is given by:

$$
Q_{n, N}(p)=\sum_{k=0}^{2 n-1}\left(\begin{array}{c}
2 N \\
k
\end{array}\right)\left(\frac{2}{p}\right)^{2 N-k}\left(\frac{p-2}{p}\right)^{k} P_{2 n-k, 2 N-k}
$$

(ii) By assuming that $(n, N)$ grows proportionally, $Q_{n, N}(p)$ tends to 0 when $\frac{n}{N}<\frac{p-1}{p}$ and tends to 1 when $\frac{n}{N}>\frac{p-1}{p}$.

Proof of Theorem 3.1. The proof of Statement ( $i)$ of Theorem 3.1 requires the introduction of the simplicity concept defined herinafter. 
Definition 3.1. Simplicity [29] A given vector $\underline{\boldsymbol{x}} \in\left[\alpha_{1}, \alpha_{p}\right]^{2 N}$ is called $k$-simple if it has exactly $k$ entries different from $\alpha_{1}$ and $\alpha_{p}$.

According to Theorem 3.1, calculating the solution uniqueness probability of the optimization problem amounts to calculating the probability that the equation $\underline{\boldsymbol{y}}=\underline{\boldsymbol{H} \boldsymbol{x}} ; \underline{\boldsymbol{x}} \in\left[\alpha_{1}, \alpha_{p}\right]^{2 N}$ has only one root. It can also be formulated as: $\underline{\boldsymbol{y}}=\underline{\boldsymbol{H} \boldsymbol{x}}, \underline{\boldsymbol{x}} \in \mathcal{P}$ with $\mathcal{P}=\left\{\underline{\boldsymbol{x}} \in \mathbb{R}^{2 N} \mid \underline{\boldsymbol{x}}=\boldsymbol{B}_{\alpha} \boldsymbol{r} ; \boldsymbol{r} \in \mathbb{R}^{4 N}, \boldsymbol{B}_{1} \boldsymbol{r}=\right.$ $\mathbf{1}_{2 N}$ and $\left.\boldsymbol{r} \geq 0\right\}$ where $\boldsymbol{B}_{\alpha}=\boldsymbol{I}_{2 N} \otimes \boldsymbol{\alpha}, \boldsymbol{\alpha}=\left[\alpha_{1}, \alpha_{p}\right]$ and $\boldsymbol{B}_{1}=\boldsymbol{I}_{2 N} \otimes \mathbf{1}_{2}^{T}$. The convex hull of this polytope which is the minimal convex set containing all the elements of $\mathcal{P}$ is the set $\left\{\boldsymbol{h} \in \mathbb{R}^{2 N} \mid h_{i}=\alpha_{1}\right.$ or $\left.h_{i}=\alpha_{p}, 1 \leq i \leq 2 N\right\}$. It contains all the vectors with entries equal to the bounds. Let $\underline{x} \in \mathbb{R}^{2 N}$ be a $k$-simple vector in $\mathcal{P}$, in other words, with exactly $k$ entries strictly different from the elements of the vectors of the convex hull of $\mathcal{P}$. Let $F$ denote the associated $k$-face of $\mathcal{P}$. Given the system $\underline{\boldsymbol{y}}=\underline{\boldsymbol{H} \boldsymbol{x}}, \underline{\boldsymbol{x}} \in \mathcal{P}$, it is showed in [29, Lemma 5.2] that the condition of solution unicity is equivalent to the condition that $\underline{\boldsymbol{H}} F$ is a $k$-face of $\underline{\boldsymbol{H}} \mathcal{P}$. In [29, Theorem 1.10], exploiting the fact that $\underline{\boldsymbol{H}}$ is completely general ${ }^{2}$, it is demonstrated that this condition is satisfied with probability $1-P_{2(N-n), 2 N-k}=P_{2 n-k, 2 N-k}$. As the elements of $\underline{\boldsymbol{x}}$ take on values with equal probability in the set $\mathcal{F}=\left\{\alpha_{1}, \alpha_{2}, \ldots, \alpha_{p}\right\}$, the probability that it is $k$-simple is $\left(\begin{array}{c}2 N \\ k\end{array}\right)\left(\frac{2}{p}\right)^{2 N-k}\left(\frac{p-2}{p}\right)^{k}$. According to Bayes' axiom the probability that $\underline{\boldsymbol{x}}$ is the unique $k$-simple solution is equal to $\left(\begin{array}{c}2 N \\ k\end{array}\right)\left(\frac{2}{p}\right)^{2 N-k}\left(\frac{p-2}{p}\right)^{k} P_{2 n-k, 2 N-k}$. Hence, the proof of Statement $(i)$. Now that Statement $(i)$ is established, the proof of Statement $(i i)$ can be obtained by following the same reasoning as in [10, Proof of Theorem 3, page 2012].

\footnotetext{
${ }^{2}$ For definition and proof see Appendix A
} 


\begin{tabular}{|r|c|c|l|}
\hline & Dimension & Cost per iteration & Total \\
\hline$\left(P_{S A, 1}\right)$ & $M N$ & $\mathcal{O}\left(M^{2} N^{2}(N+n)\right)$ & $\mathcal{O}\left(M^{2} N^{2}(N+n)^{3 / 2}\right)$ \\
\hline$\left(P_{H S A, 1}\right)$ & $\log _{2}(M) N$ & $\mathcal{O}\left(\left(\log _{2}(M)\right)^{2} N^{2}(N+n)\right)$ & $\mathcal{O}\left(\left(\log _{2}(M)\right)^{2} N^{2}(N+n)^{3 / 2}\right)$ \\
\hline$\left(P_{\infty}\right)$ & $2 N$ & $\mathcal{O}\left(N^{2}(N+n)\right)$ & $\mathcal{O}\left(N^{2}(N+n)^{3 / 2}\right)$ \\
\hline$\left(P_{S I, 1}\right)$ & $2 N$ & $\mathcal{O}\left(N^{2}(N+n)\right)$ & $\mathcal{O}\left(N^{2}(N+n)^{3 / 2}\right)$ \\
\hline
\end{tabular}

Table 1: Computation cost with the interior point method.

\subsection{Complexity Analysis}

The interest of the proposed detection scheme comes from its complexity order. The CVX toolbox relies on the interior point method whose complexity is a function of the number of constraints and the dimension of the searched vector $[30,31]$. A convex optimization problem defined over $\mathbb{R}^{m}$ subject to $d$ constraints requires, in the worst case, $\mathcal{O}(\sqrt{d})$ iterations for a computation cost order of $\mathcal{O}\left(m^{2} d\right)$ per iteration and yields a total computation cost order equal to $\mathcal{O}\left(m^{2} d^{3 / 2}\right)$ [32]. Applied to the different convex optimization problems dealt with in this section, we obtain the computation costs reported in Table I. According to these estimations, the half-sparse decomposition enables to reduce the computation cost by $\left(\frac{p}{\log _{2}(p)}\right)^{2}$. However, in the case of binary alphabets, $\left(P_{\infty}\right)$ is the most interested because its complexity order is the lowest. For higher-size alphabets, $\left(P_{\infty}\right)$ does not apply and $\left(P_{S I, 1}\right)$ becomes the most relevant problem to solve. Its computation cost is the same as $\left(P_{\infty}\right)$ and keeps constant whatever the alphabet size.

\subsection{Simulation results}




\begin{tabular}{|l|l|}
\hline Parameters & Value \\
\hline \hline Mixing matrix $\boldsymbol{H}$ & $H_{i j} \sim \operatorname{eN}(0,1)$ i.i.d for $\forall i, j$ \\
\hline Mixing matrix knowledge & Perfect \\
\hline Alphabet size $p$ & $p \in\{2,4,8\}$ \\
\hline Alphabet construction & $\mathcal{F}=\{ \pm(2 k-1): k=\{1,2, \ldots, p / 2\}\}$ \\
\hline Number of sources $N$ & $N \in\{64,128,256\}$ \\
\hline Monte Carlo iteration number & 1000 \\
\hline Matlab toolbox & CVX toolbox \\
\hline
\end{tabular}

Table 2: Simulation setup for Section 3.3.

The following simulation results illustrate the theoretical framework exposed above. The experimental setup is common to all simulations. We use even values of $p$ and choose $\mathcal{F}=\{ \pm(2 k-1): k=\{1,2, \ldots, p / 2\}\}$. For each simulation, we fix $N \in\{64,128,256\}$ and make $n$ vary so as to assess a significant number of values for ratio $n / N$. For each pair $(n, N), 1000$ iterations are carried out. For each iteration, we generate a realization of the complex-valued generic random matrix of size $n \times N$. The matrix coefficients are independent and identically Gaussian distributed with zero mean and unit variance. We then transform it in a real-valued formulation with size $2 n \times 2 N$. We generate $\underline{\boldsymbol{x}}$ with $2 N$ entries drawn uniformly from $\mathcal{F}$. We solve the optimization problem by using the Matlab CVX toolbox [30]. The simulation results are obtained by using a PC equipped with Linux Ubuntu 14.04 OS, Intel Core i3-2350M processor $(2.3 \mathrm{GHz})$ and 8GB RAM. A solution $\underline{\hat{\boldsymbol{x}}}$ is returned and we assume that the recovery is correct if the relative 
error $\frac{\|\underline{\hat{\boldsymbol{x}}}-\underline{\boldsymbol{x}}\|_{2}}{\|\underline{\boldsymbol{x}}\|_{2}}$ is less than $10^{-6}$. The simulation setup is summarized in Table 2 .

Fig. 1 is the phase diagram in the case $p=2$ for the proposed simplicitybased approach. The simulated successful recovery probability corroborates Theorem 3.1 and it coincides with the analytical expression. In particular, we observe that the breakpoint occurs when $n / N=1 / 2$ with a successful recovery probability equal to one half, as established theoretically. We also recall that the proposed simplicity-based recovery method performs the same as Mangasarian approach.

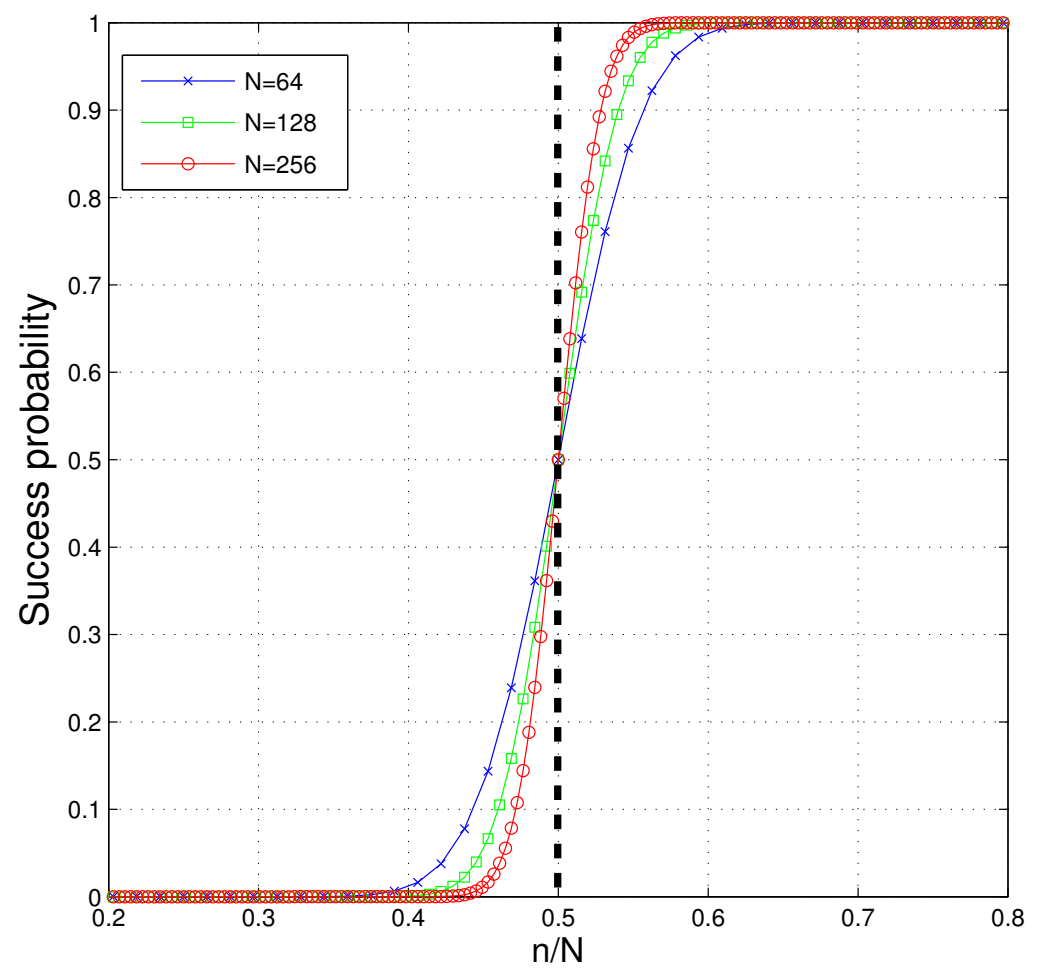

Figure 1: Phase diagrams of the proposed method, for $p=2$ and $N \in\{64,128,256\}$. 


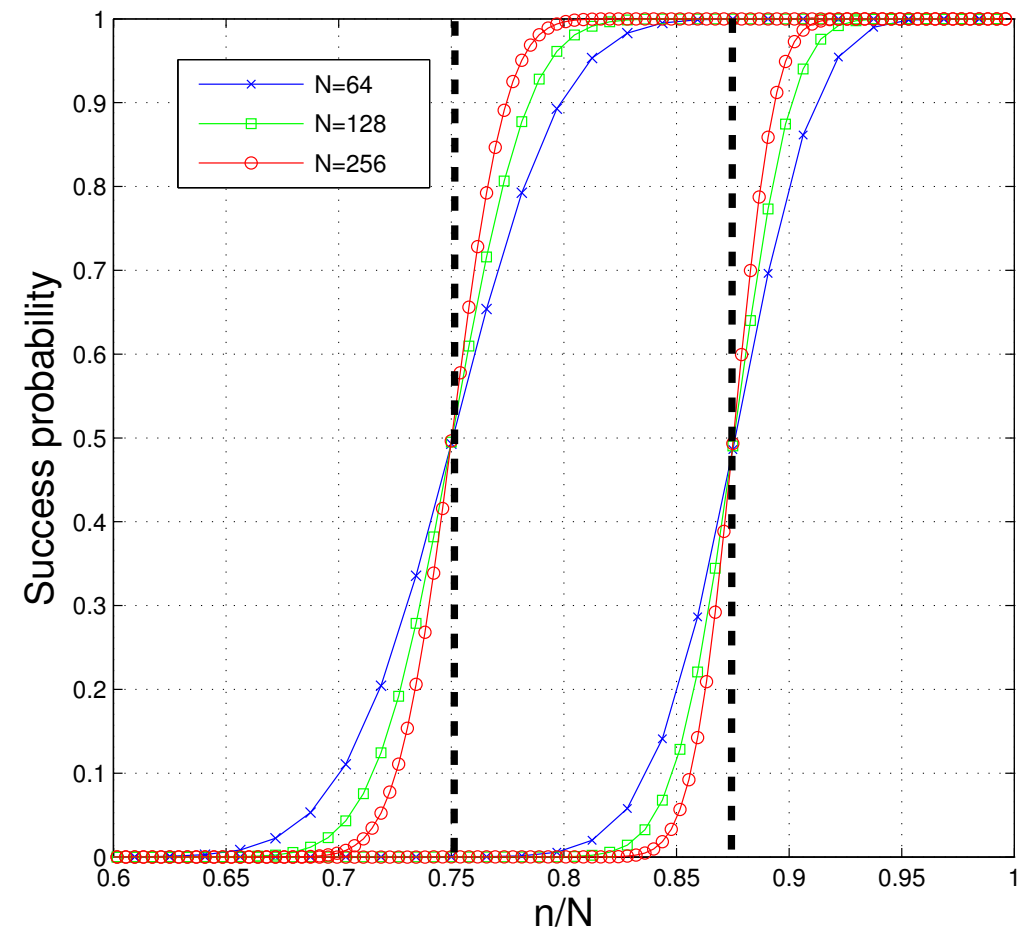

Figure 2: Phase diagrams of the proposed method, for $p=4, p=8$ and $N \in\{64,128,256\}$.

We now address the case $p>2$, for which the Mangasarian et al. approach is not applicable. Fig. 2 provides the phase diagrams of the proposed approach $\left(P_{S I, 1}\right)$ for $p=4$ and $p=8$ and different values of $N$. The simulated successful recovery probability coincides with the analytical expression. The simulation results confirm the theoretical study presented above. The breakpoint occurs when $\frac{n}{N}$ equals the value $\frac{p-1}{p}$, that is to say $\frac{3}{4}$ for $p=4$ and $\frac{7}{8}$ for $p=8$. 


\section{Application of the simplicity principle to noisy massive MIMO transmission}

\subsection{Proposed method definition and theoretical analysis}

We consider in this section the noisy version of the problem that is to say $\boldsymbol{y}=\boldsymbol{H} \boldsymbol{x}+\boldsymbol{\zeta}$. We propose to recover the vector $\boldsymbol{x}$ thanks to the previously introduced decomposition that exploits the fact that the real and imaginary parts of the symbols belong to the interval $\left[\alpha_{1}, \alpha_{p}\right]$.

Considering the proposed real-valued formulation, the Maximum Likelihood [ML] problem reads

$$
\left(P_{M L, 2}\right): \underset{\underline{\boldsymbol{x}}}{\arg \min }\|\underline{\boldsymbol{y}}-\underline{\boldsymbol{H} \boldsymbol{x}}\|_{2} \quad \text { subject to } \quad \underline{\boldsymbol{x}} \in \mathcal{F}^{2 N} .
$$

As mentioned above this problem is NP-hard with high order of complexity. To circumvent this problem, we proceed in the same way as in the noisefree case and based on the Theorem 3.1 we propose to solve the following optimization problem:

$$
\left(P_{S I, 2}\right): \underset{r}{\arg \min }\left\|\underline{\boldsymbol{y}}-\underline{\boldsymbol{H}} \boldsymbol{B}_{\alpha} \boldsymbol{r}\right\|_{2} \quad \text { subject to } \quad \boldsymbol{B}_{1} \boldsymbol{r}=\mathbf{1}_{2 N}, \quad \boldsymbol{r} \geq 0 .
$$

To evaluate its performance, we search for the conditions for a stationary point and we investigate the statistical distribution of the detection output. Let us define the necessary sets used to establish the analytical results.

Definition 4.1. Let $\Omega$ the set of active constraints defined by $\Omega=\left\{i \mid \underline{x}_{i}=\alpha_{1}\right.$ or $\left.\underline{x}_{i}=\alpha_{p}\right\}$ and $\Lambda$ the set of binding constraints defined by $\Lambda=\left\{i \mid \underline{x}_{i}=\alpha_{1}\right.$ and $\left\{\underline{\boldsymbol{H}}^{T}(\underline{\boldsymbol{H} \hat{\boldsymbol{x}}}-\underline{\boldsymbol{y}})\right\}_{i} \geq 0$ or $\underline{x}_{i}=\alpha_{p}$ and $\left.\left\{\underline{\boldsymbol{H}}^{T}(\underline{\boldsymbol{H} \hat{\boldsymbol{x}}}-\underline{\boldsymbol{y}})\right\}_{i} \leq 0\right\}$. The cardinality of $\Lambda$ defines the simplicity order of the searched vector. 
The complementary set $\bar{\Lambda}$ corresponds to the non-binding constraint set. Its cardinality is denoted by $\mathrm{e}=\operatorname{Card}(\bar{\Lambda})$.

The following theorem gives the conditions for a solution to be a stationary point for $\left(P_{S I, 2}\right)$.

\section{Theorem 4.1. Stationary point condition [33]}

$\hat{\boldsymbol{r}}$ is a stationary point for $\left(P_{S I, 2}\right)$ (a point satisfying the first order necessary conditions for optimality $)$ if and only if $\underline{\hat{\boldsymbol{x}}}=\boldsymbol{B}_{\alpha} \hat{\boldsymbol{r}}$ is feasible and $\left\{\underline{\boldsymbol{H}}^{T}(\underline{\boldsymbol{H} \hat{\boldsymbol{x}}}-\right.$ $\underline{\boldsymbol{y}})\}_{\bar{\Lambda}}=\mathbf{0}$.

The output solution verifies the stationary point condition. In order to define the theoretical performance and to enhance the recovery performance either by an iterative scheme or by the addition of a forward error correction code soft-decision decoder, we need the probability density function of the detector output. The following theorem defines its theoretical statistical distribution.

\section{Theorem 4.2. Statistical distribution of the detection output}

Let $\hat{\boldsymbol{r}}$ the solution of $\left(P_{S I, 2}\right)$. Then the components of $\underline{\hat{\boldsymbol{x}}}=\boldsymbol{B}_{\alpha} \hat{\boldsymbol{r}}$ follow a censored normal distribution given by

$$
f_{\underline{\underline{x}}_{k}}(x)=\frac{1}{p} \sum_{j=1}^{p} f_{\underline{\hat{x}}_{k} \mid x_{k}=\alpha_{j}}(x),
$$

with

$$
\begin{aligned}
& f_{\underline{\hat{x}}_{k} \mid \underline{x}_{k}=\alpha_{j}}(x)=\left(\frac{1}{2} \operatorname{erfc}\left(\frac{\alpha_{j}-\alpha_{1}}{\sqrt{2} \sigma_{\underline{\hat{x}}}}\right) \delta_{\alpha_{1}}(x)\right. \\
& +\frac{1}{2} \operatorname{erfc}\left(\frac{\alpha_{p}-\alpha_{j}}{\sqrt{2} \sigma_{\underline{\hat{x}}}}\right) \delta_{\alpha_{p}}(x) \\
& \left.+\frac{1}{\sqrt{2 \pi} \sigma_{\underline{\underline{\hat{x}}}}} \exp \left(-\frac{\left(x-\alpha_{j}\right)^{2}}{2 \sigma_{\underline{\underline{x}}}^{2}}\right) \mathbb{1}_{\left[\alpha_{1}, \alpha_{p}\right]}(x)\right)
\end{aligned}
$$


and

$$
\sigma_{\underline{\hat{x}}}^{2}=\sum_{k=0}^{2 n-2}\left(\begin{array}{c}
2 N \\
k
\end{array}\right)\left(\frac{1}{p}\right)^{2 N-k}\left(\frac{p-1}{p}\right)^{k} \frac{2 n \sigma^{2}}{2 n-k-1},
$$

where $\sigma^{2}=\mathbb{E}\left[\underline{\zeta}_{i}^{2}\right], \forall i=1, \ldots, 2 n$.

Proof of Theorem 4.2. Let us consider $\hat{\boldsymbol{r}}$ a stationary point of problem $\left(P_{S I, 2}\right)$. Then $\underline{\hat{\boldsymbol{x}}}=\boldsymbol{B}_{\alpha} \hat{\boldsymbol{r}}$ is feasible and $\left\{\underline{\boldsymbol{H}}^{T}(\underline{\boldsymbol{H} \hat{\boldsymbol{x}}}-\underline{\boldsymbol{y}})\right\}_{\bar{\Lambda}}=\mathbf{0}$ (see Theorem 4.1). Let $\underline{\boldsymbol{H}}_{\bar{\Lambda}}=\left[\underline{\boldsymbol{H}}_{:, k}\right]_{k \in \bar{\Lambda}}$ with $\underline{\boldsymbol{H}}_{:, k}$ the $k^{\text {th }}$ column of $\underline{\boldsymbol{H}}$. We first assume that $\mathcal{C}=\operatorname{Card}(\bar{\Lambda})$ satisfies $\mathcal{C}<2 n-1$ so that the Moore-Penrose pseudo-inverse of $\underline{\boldsymbol{H}}_{\bar{\Lambda}}$ exists and equals $\underline{\boldsymbol{H}}_{\bar{\Lambda}}^{\dagger}=\left(\underline{\boldsymbol{H}}_{\bar{\Lambda}}^{T} \underline{\boldsymbol{H}}_{\bar{\Lambda}}\right)^{-1} \underline{\boldsymbol{H}}_{\bar{\Lambda}}^{T}$. Therefore, the restriction of $\underline{\hat{\boldsymbol{x}}}$ to the index set $\bar{\Lambda}$ reads

$$
\underline{\hat{\boldsymbol{x}}}_{\bar{\Lambda}}=\underline{\boldsymbol{H}}_{\bar{\Lambda}}^{\dagger}\left(\underline{\boldsymbol{y}}-\underline{\boldsymbol{H}}_{\Lambda} \underline{\hat{\boldsymbol{x}}}_{\Lambda}\right) .
$$

From Eq. (20) and exploiting the property that the set of binding constraints $\Lambda$ can be seen as the set of indexes of entries of $\underline{\boldsymbol{x}}$ which were correctly estimated, according to the central limit theorem, given $\underline{\boldsymbol{x}}$ and $\mathcal{C}, \underline{\hat{\boldsymbol{x}}}_{\bar{\Lambda}}$ is normally distributed with mean $\underline{\boldsymbol{x}}_{\bar{\Lambda}}$. To compute the covariance matrix $\boldsymbol{\Sigma}_{\underline{\hat{\boldsymbol{x}}}_{\overline{\boldsymbol{\Lambda}}}}$ we exploit the fact that the number of non-binding constraints $\mathcal{C}$ is a random variable. Therefore, $\boldsymbol{\Sigma}_{\underline{\hat{\boldsymbol{x}}}_{\bar{\Lambda}}}$ is given by:

$$
\underline{\boldsymbol{\Sigma}}_{\underline{\hat{\boldsymbol{x}}}_{\bar{\Lambda}}}=\mathbb{E}\left[\mathbb{E}\left[\left(\underline{\hat{\boldsymbol{x}}}_{\bar{\Lambda}}-\mathbb{E}\left[\underline{\hat{\boldsymbol{x}}}_{\bar{\Lambda}}\right]\right)\left(\underline{\hat{\boldsymbol{x}}}_{\bar{\Lambda}}-\mathbb{E}\left[\underline{\hat{\boldsymbol{x}}}_{\bar{\Lambda}}\right]\right)^{T} \mid \mathcal{C}=k\right]\right],
$$

with

$$
\begin{aligned}
\mathbb{E}\left[\left(\underline{\hat{\boldsymbol{x}}}_{\bar{\Lambda}}-\mathbb{E}\left[\underline{\hat{\boldsymbol{x}}}_{\bar{\Lambda}}\right]\right)\left(\underline{\hat{\boldsymbol{x}}}_{\bar{\Lambda}}-\mathbb{E}\left[\underline{\hat{\boldsymbol{x}}}_{\bar{\Lambda}}\right]\right)^{T} \mid \mathcal{C}=k\right] & =\sigma^{2} \mathbb{E}\left[\left(\underline{\boldsymbol{H}}_{\bar{\Lambda}}^{T} \underline{\boldsymbol{H}}_{\bar{\Lambda}}\right)^{-1} \mid \mathcal{C}=k\right] \\
& =\sigma^{2} \frac{2 n}{2 n-k-1} \boldsymbol{I}_{k},
\end{aligned}
$$

where we have used that, given $\mathcal{C}=k$, the matrix $\left(\underline{\boldsymbol{H}}_{\bar{\Lambda}}^{T} \underline{\boldsymbol{H}}_{\bar{\Lambda}}\right)^{-1}$ follows an inverse Wishart distribution and then $\mathbb{E}\left[\left(\underline{\boldsymbol{H}}_{\bar{\Lambda}}^{T} \underline{\boldsymbol{H}}_{\bar{\Lambda}}\right)^{-1} \mid \mathrm{C}=k\right]=\frac{2 n}{2 n-k-1} \boldsymbol{I}_{k}$ (see [34]). The distribution of $\mathcal{C}$ is provided by the following Proposition 4.1. 
Proposition 4.1. The number of non binding constraints introduced in Definition 4.1 follows the binomial distribution with parameters $2 N$ and $\frac{p-1}{p}$ :

$$
\mathcal{C}=\operatorname{Card}(\bar{\Lambda}) \sim \mathcal{B}\left(2 N, \frac{p-1}{p}\right)
$$

The proof of Proposition 4.1 is given in Appendix B. From Proposition 4.1 , we observe that the probability of event $\mathcal{C} \geq 2 n-1$ is not significant and these events will thus be neglected in the computation of $\boldsymbol{\Sigma}_{\hat{\boldsymbol{x}}_{\bar{\Lambda}}}$. We then obtain

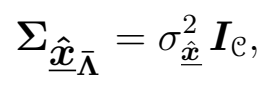

with

$\sigma_{\underline{\underline{\hat{x}}}}^{2}=\sum_{k=0}^{2 n-2} \operatorname{Pr}(\mathcal{C}=k) \frac{2 n \sigma^{2}}{2 n-k-1}=\sum_{k=0}^{2 n-2}\left(\begin{array}{c}2 N \\ k\end{array}\right)\left(\frac{1}{p}\right)^{2 N-k}\left(\frac{p-1}{p}\right)^{k} \frac{2 n \sigma^{2}}{2 n-k-1}$.

Finally, the constraints of $\left(P_{S I, 2}\right)$ impose that $\underline{\hat{x}}_{i} \in\left[\alpha_{1}, \alpha_{p}\right]$ and we deduce that, given $\underline{\boldsymbol{x}}$, the components of $\underline{\hat{\boldsymbol{x}}}$ corresponding to the non-binding constraints follow a truncated normal distribution with mean $\underline{\boldsymbol{x}}$ and variance $\sigma_{\underline{\hat{x}}}^{2}$

As for the components of $\underline{\hat{\boldsymbol{x}}}$ corresponding to the binding constraints, they satisfy either $\underline{x}_{i}=\alpha_{1}$ and $\left\{\underline{\boldsymbol{H}}^{T}(\underline{\boldsymbol{H} \hat{\boldsymbol{x}}}-\underline{\boldsymbol{y}})\right\}_{i} \geq 0$, or $\underline{x}_{i}=\alpha_{p}$ and $\left\{\underline{\boldsymbol{H}}^{T}(\underline{\boldsymbol{H} \hat{\boldsymbol{x}}}-\right.$ $\left.\underline{\boldsymbol{y}})\}_{i} \leq 0\right\}$. We thus conclude that they follow a binary distribution with probability $\frac{1}{2 p}$ (see Appendix B for the justification of $\operatorname{Pr}\left(\left\{\underline{\boldsymbol{H}}^{T}(\underline{\boldsymbol{H} \hat{\boldsymbol{x}}}-\underline{\boldsymbol{y}})\right\}_{i} \geq\right.$ $\left.0)=\frac{1}{2}\right)$.

Consequently, the conditional distribution of $\underline{\hat{x}}_{k}$ given $x_{k}$ reads

$$
\begin{aligned}
& f_{\underline{\hat{x}}_{k} \mid \underline{x}_{k}=\alpha_{j}}(x)=\left(\frac{1}{2} \operatorname{erfc}\left(\frac{\alpha_{j}-\alpha_{1}}{\sqrt{2} \sigma_{\underline{\hat{x}}}}\right) \delta_{\alpha_{1}}(x)+\frac{1}{2} \operatorname{erfc}\left(\frac{\alpha_{p}-\alpha_{j}}{\sqrt{2} \sigma_{\underline{\hat{x}}}}\right) \delta_{\alpha_{p}}(x)\right. \\
& \left.+\frac{1}{\sqrt{2 \pi} \sigma_{\underline{\hat{x}}}} \exp \left(-\frac{\left(x-\alpha_{j}\right)^{2}}{2 \sigma_{\underline{\hat{x}}}^{2}}\right) 1_{\left[\alpha_{1}, \alpha_{p}\right]}(x)\right)
\end{aligned}
$$


As demonstrated, we can see the distribution of the output vector as a mix of a binary distribution due to the simplicity of the vector and a truncated normal distribution with variance depending on the system dimensions and the noise variance. Depending on the system dimensions, the exact variance computation may be too complex in practice. The following lemma provides an approximation of the variance which can be simply calculated. Its accuracy will be studied in the simulation section.

\section{Lemma 4.1. Variance approximation}

Let $\hat{\boldsymbol{r}}$ the solution of $\left(P_{S I, 2}\right)$. The variance of the output vector $\underline{\hat{\boldsymbol{x}}}=\boldsymbol{B}_{\alpha} \hat{\boldsymbol{r}}$ can be approximated for $n \geq N\left(\frac{p-1}{p}\right)+1$ as

$$
\sigma_{\underline{\hat{\boldsymbol{x}}}}^{2} \approx \frac{2 n \sigma^{2}}{2 n-2 N\left(\frac{p-1}{p}\right)-1} .
$$

From the statistical distribution of the detection output and by exploiting the general results available in [35], a lower bound of the symbol error probability can be obtained. This bound is asymptotically reached when the SNR gets high, which provides an approximation of the symbol error probability. It can be used to predict performance without simulating the whole system and its accuracy will be checked in the simulation part.

\section{Theorem 4.3. Symbol Error Probability upper-bound}

The symbol error probability in the case of a M-ary QAM constellation can be upper-bounded by:

$$
P_{s} \leq \frac{1}{2 p} \sum_{k=1}^{p} \sum_{\substack{j=1 \\ j \neq k}}^{p} \operatorname{erfc}\left(\frac{\alpha_{j}-\alpha_{k}}{2 \sqrt{2} \sigma_{\underline{\underline{x}}}}\right)+\frac{p-1}{2 p} \sum_{i=1}^{p} \operatorname{erfc}\left(\frac{\alpha_{p}-\alpha_{i}}{\sqrt{2} \sigma_{\underline{\underline{x}}}}\right) .
$$


This upper bound can be used as a tight approximation of the symbol error probability. For high SNR, the symbol error probability can be further approximated by:

$$
P_{s} \approx \frac{p-1}{p} \operatorname{erfc}\left(\frac{\alpha_{2}-\alpha_{1}}{2 \sqrt{2} \sigma_{\underline{\hat{x}}}}\right) .
$$

\subsection{Complexity Analysis}

Table II summarizes the complexity order of the decomposition-based detectors including the proposed one referred to as $\left(P_{S I, 2}\right)$, the MMSE, the MMSE-SIC, the MMSE-SIC-LR, and the SD detector. The SD detector is a high-complexity detector especially when the modulation order or the number of antennas increase, it is the least cost efficient. The MMSE-based detector consists of one matrix inversion and some matrix multiplications and additions. The MMSE-SIC adds some order of complexity. According to the complexity analysis in [36], the additional complexity order involved in the MMSE-SIC-LR due to lattice reduction is equal to $\mathcal{O}\left(N^{2} \log B\right)$ where $B$ is is the norm of the longest basis vector. In the case of determined MIMO systems, $\left(P_{S I, 2}\right)$ achieves the same order of complexity compared to the MMSE-based methods. As $\left(P_{S I, 2}\right)$ and $\left(P_{S A, 2}\right)$ are optimized with the same algorithm (interior point method) and in order to show the complexity reduction, we plotted the run-time of both detectors for high-order modulations. In Fig. 3, the 16-QAM modulation is considered with $N=64$. We show that for $\frac{n}{N}=0.8$ (underdetermined system), the complexity is divided by two. In Fig. 4 the run-time is plotted for 64-QAM and $N=64$. We observe that the gain is even higher than for 16-QAM and the complexity order of the proposed algorithm is similar in both modulation cases. These results are in accordance with Table 3. 


\begin{tabular}{|c|c|c|c|}
\hline & Iteration number & Cost per iteration & Total \\
\hline MMSE & 1 & $\mathcal{O}\left(N^{3}\right)$ & $\mathcal{O}\left(N^{3}\right)$ \\
\hline MMSE-SIC & 1 & $\begin{array}{c}\mathcal{O}\left(N^{3}\right)+\mathcal{O}\left(M N^{2}\right) \\
+\mathcal{O}\left(M^{2} N\right)\end{array}$ & $\begin{array}{l}\mathcal{O}\left(N^{3}\right)+\mathcal{O}\left(M N^{2}\right) \\
+\mathcal{O}\left(M^{2} N\right)\end{array}$ \\
\hline MMSE-SIC-LR & 1 & $\begin{array}{c}\mathcal{O}\left(N^{3}\right)+\mathcal{O}\left(M N^{2}\right) \\
+\mathcal{O}\left(M^{2} N\right)+\mathcal{O}\left(N^{2} \log B\right)\end{array}$ & $\begin{array}{l}\mathcal{O}\left(N^{3}\right)+\mathcal{O}\left(M N^{2}\right) \\
+\mathcal{O}\left(M^{2} N\right)+\mathcal{O}\left(N^{2} \log B\right)\end{array}$ \\
\hline$\left(P_{S I, 2}\right)$ & $\mathcal{O}(\sqrt{N})$ & $\mathcal{O}\left(N^{2.5}\right)$ & $\mathcal{O}\left(N^{3}\right)$ \\
\hline$\left(P_{H S A, 2}\right)$ & $\mathcal{O}\left(\sqrt{2 \log _{2}(M) N}\right)$ & $\mathcal{O}\left(N^{2.5}\right)$ & $\mathcal{O}\left(\sqrt{2 \log _{2}(M)} N^{3}\right)$ \\
\hline$\left(P_{S A, 2}\right)$ & $\mathcal{O}(\sqrt{M N})$ & $\mathcal{O}\left(N^{2.5}\right)$ & $\mathcal{O}\left(\sqrt{M} N^{3}\right)$ \\
\hline$S D$ & 1 & $\mathcal{O}\left(\sqrt{M^{N}}\right)$ & $\mathcal{O}\left(\sqrt{M^{N}}\right)$ \\
\hline
\end{tabular}

Table 3: Computational cost with the interior point method.

\subsection{Simulation results: perfect channel estimation assumption}

In this section, we assume perfect channel state information and we evaluate the error rate achieved by the proposed detector based on $\left(P_{S I, 2}\right)$. We consider $n \times N$ MIMO systems, where $N$ and $n$ are the number of symbols to be recovered and the number of observations, respectively. $\boldsymbol{H}$ is a complex valued generic random matrix of size $n \times N$. We transform it in a real-valued formulation $\underline{\boldsymbol{H}}$ with size $2 n \times 2 N$. The channel coefficients are independent and identically Gaussian distributed with zero mean and unit variance, and the data symbols belong to a finite QAM alphabet. We use the Matlab CVX toolbox again. The quadratic minimization problem $\left(P_{S I, 2}\right)$ is solved by the Gurobi optimizer [37]. The simulation setup is summarized in Table 4. 


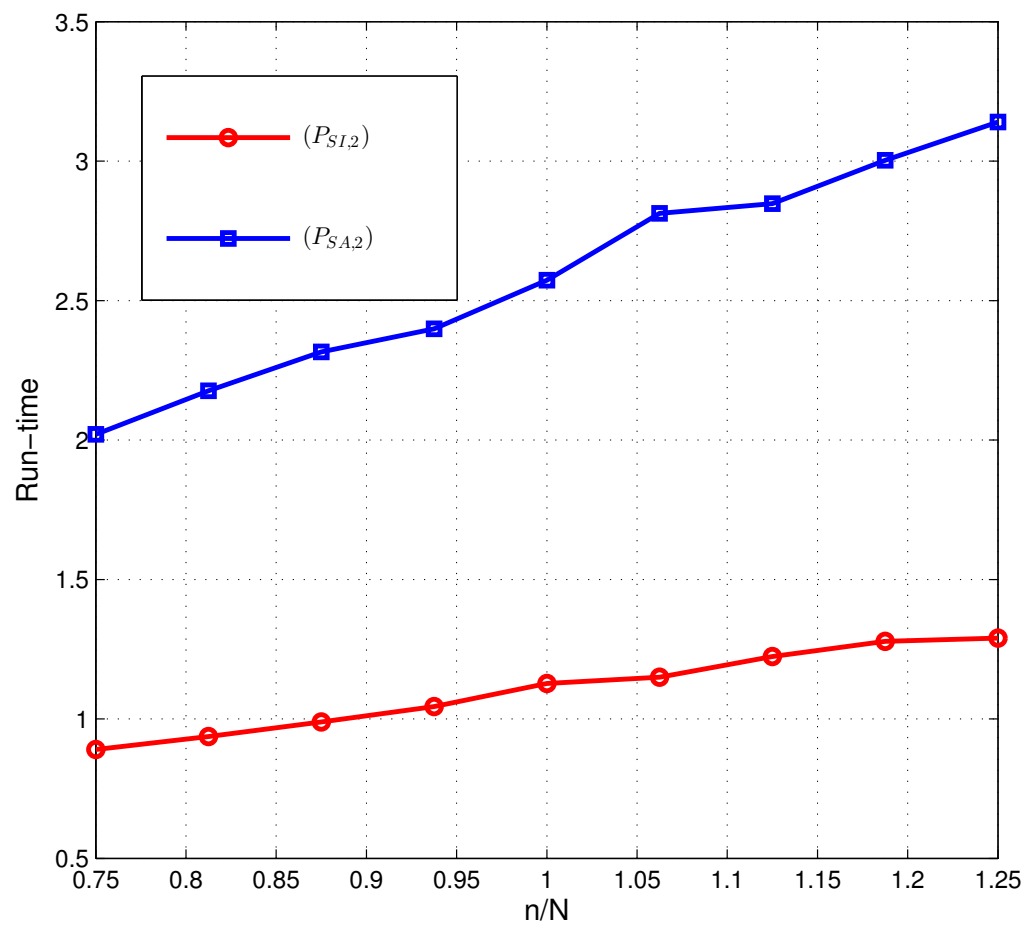

Figure 3: Run-time Comparison for 16-QAM, $N=64$ and $\mathrm{SNR}=25 \mathrm{~dB}$.

\subsubsection{Comparison of the simulation results with the theoretical analysis}

We first check that the simulated histogram of detection output is in accordance with the theoretical statistical distribution given in Theorem 4.2 and Lemma 4.1. Fig. 5, Fig. 6, Fig. 7 and Fig. 8 give the results for a $64 \times 64$ system $(n=N=64)$ and 16-QAM with $\mathrm{SNR}=15 \mathrm{~dB}$ and $\mathrm{SNR}=30 \mathrm{~dB}$ and 64 -QAM with $\mathrm{SNR}=20 \mathrm{~dB}$ and $\mathrm{SNR}=35 \mathrm{~dB}$ respectively. From these figures, we observe that the theoretical distribution (exact as well as approximate) coincides with the simulated histogram for both low and high SNRs and different modulation orders. 


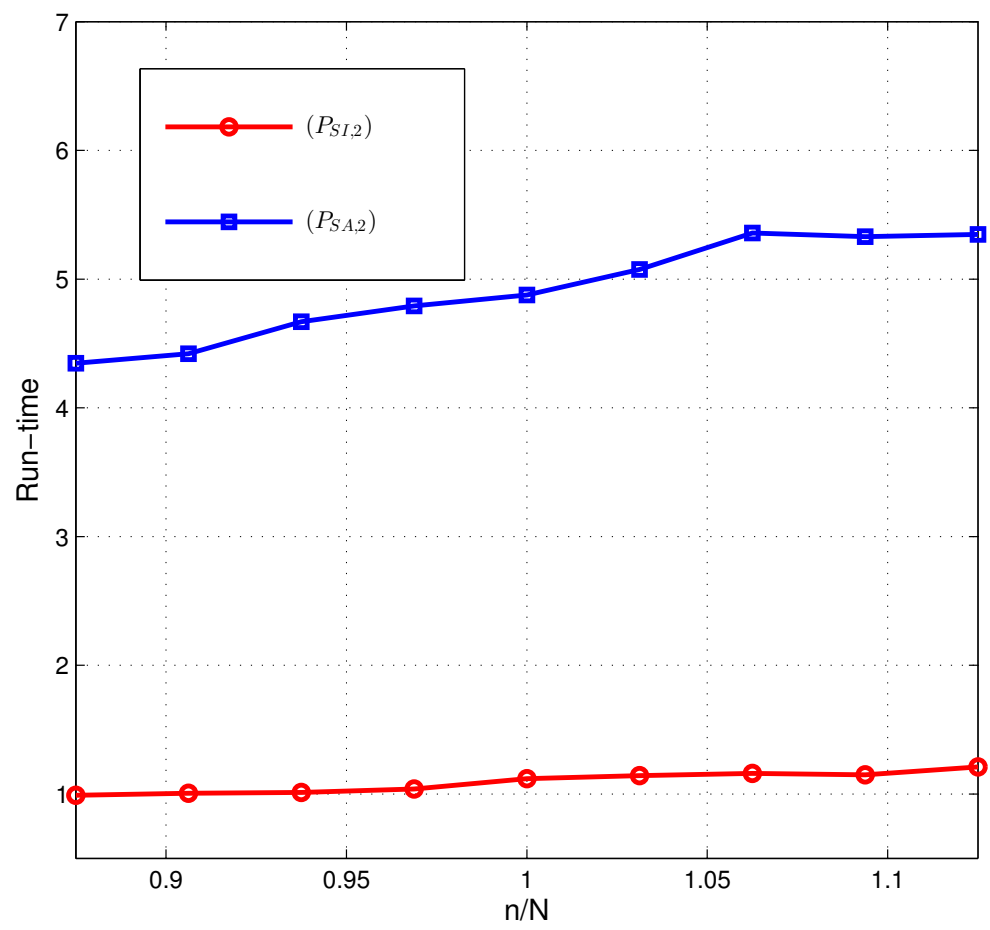

Figure 4: Run-time Comparison for $64-\mathrm{QAM}, N=64$ and $\mathrm{SNR}=35 \mathrm{~dB}$.

In Fig. 9, both simulated SER and theoretical symbol error probability approximations are plotted for $n=N=32$ and 16-QAM. We observe that the approximation given by Eq. (25) coincides with the simulated SER. The other one given by Eq. (26) is slightly more optimistic than simulated SER for very low SNR values. These observations validate the theoretical analysis.

\subsubsection{Comparison with the SD (underdetermined case)}

Fig. 10 shows the performance of the proposed scheme $\left(P_{S I, 2}\right)$ for the underdetermined MIMO system of size $24 \times 18$ with 4 -QAM. We observe 


\begin{tabular}{|l|l|}
\hline Parameters & Value \\
\hline \hline Mixing matrix $\boldsymbol{H}$ & $H_{i j} \sim \operatorname{eN}(0,1)$ i.i.d for $\forall i, j$ \\
\hline Mixing matrix knowledge & Perfect \\
\hline QAM modulation order $M$ & $M \in\{4,16\}$ \\
\hline Number of sources $N$ & $N \in\{24,32,64\}$ \\
\hline Number of observations $n$ & $n \in\{18,32,64\}$ \\
\hline Matlab toolbox & CVX toolbox \\
\hline
\end{tabular}

Table 4: Simulation setup for Section 4.3.

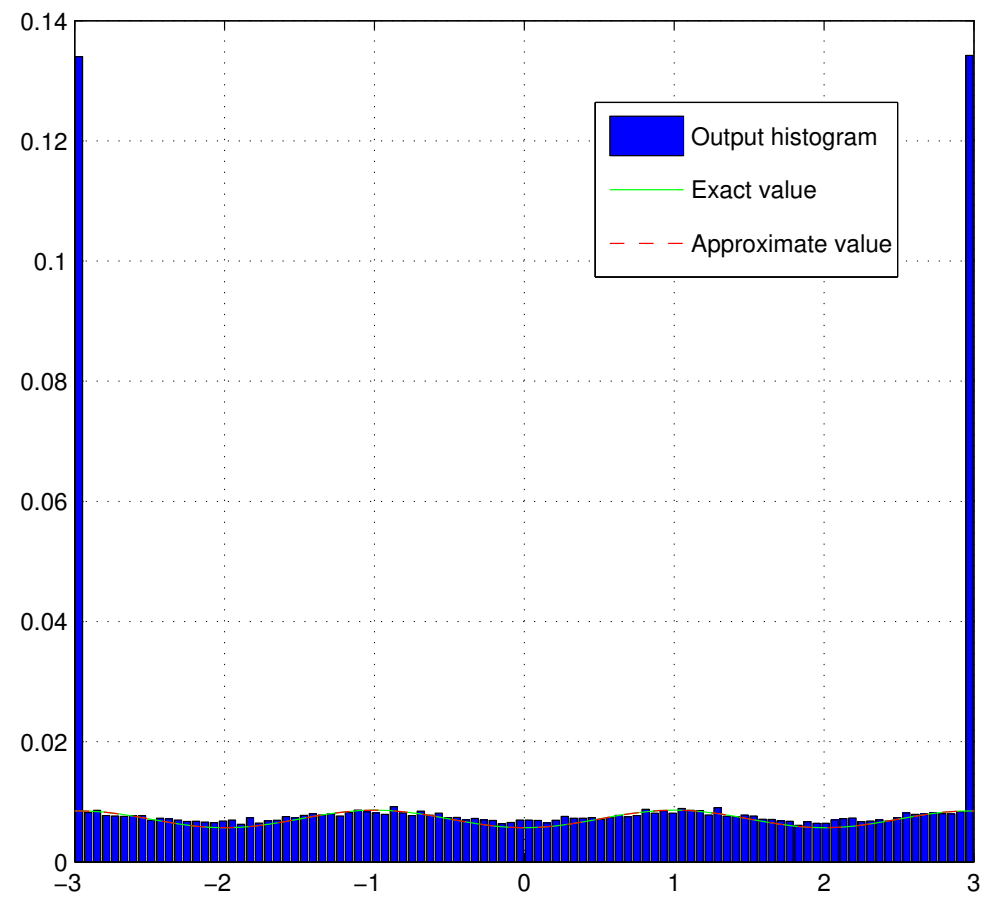

Figure 5: Output statistics for $64 \times 64$ systems with 16 -QAM and SNR=15dB (low SNR). 


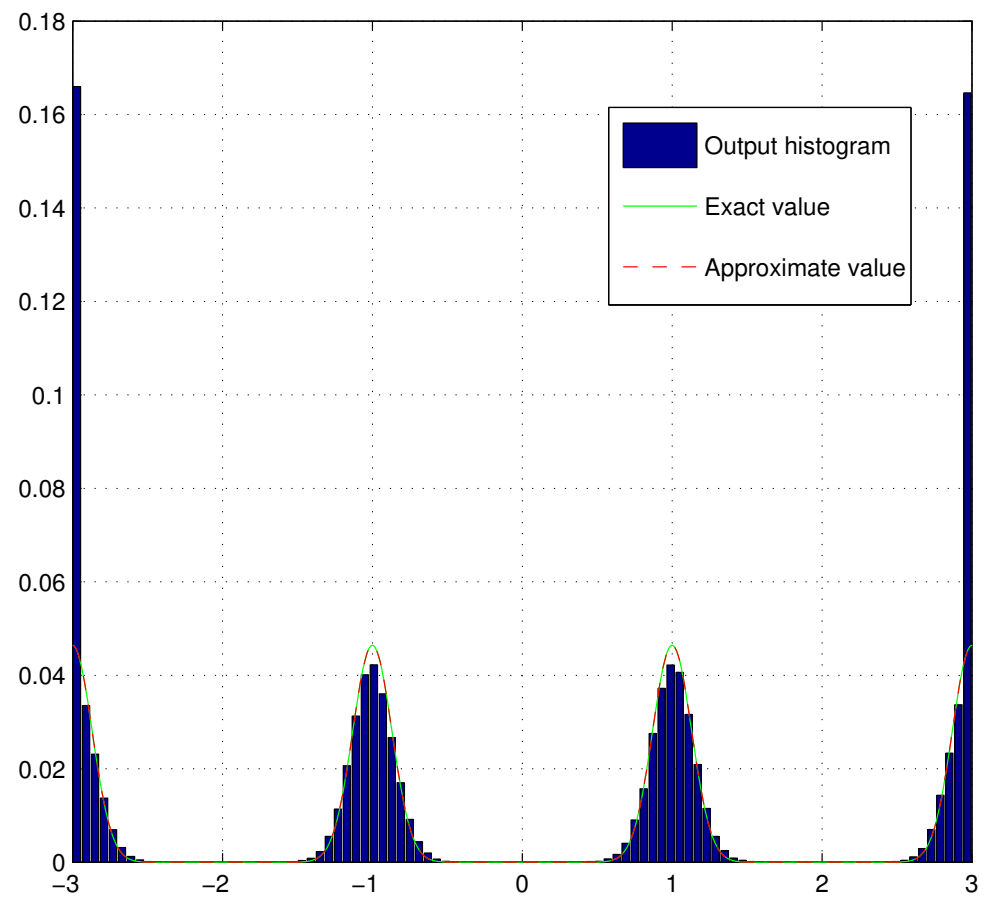

Figure 6: Output statistics for $64 \times 64$ systems with 16 -QAM and SNR=30dB (high SNR).

that it achieves a BER under $10^{-3}$ for the SNR values higher than $20 \mathrm{~dB}$. Beyond $8 \mathrm{~dB}$, the sphere decoder [26] outperforms the proposed scheme, e.g., at BER $10^{-3}$, the gain is about $6.3 \mathrm{~dB}$. However as the MIMO system dimensions increase, the SD computation cost will rapidly become too high to be implemented in practice, making the SD inadequate for large-scale MIMO applications.

\subsubsection{Comparison with MMSE-based detection schemes (determined case)}

Fig. 11 considers a determined system with $N=n=64$ and 4-QAM. We compare simplicity-based detection $\left(P_{S I, 2}\right)$ to MMSE, MMSE-SIC and 


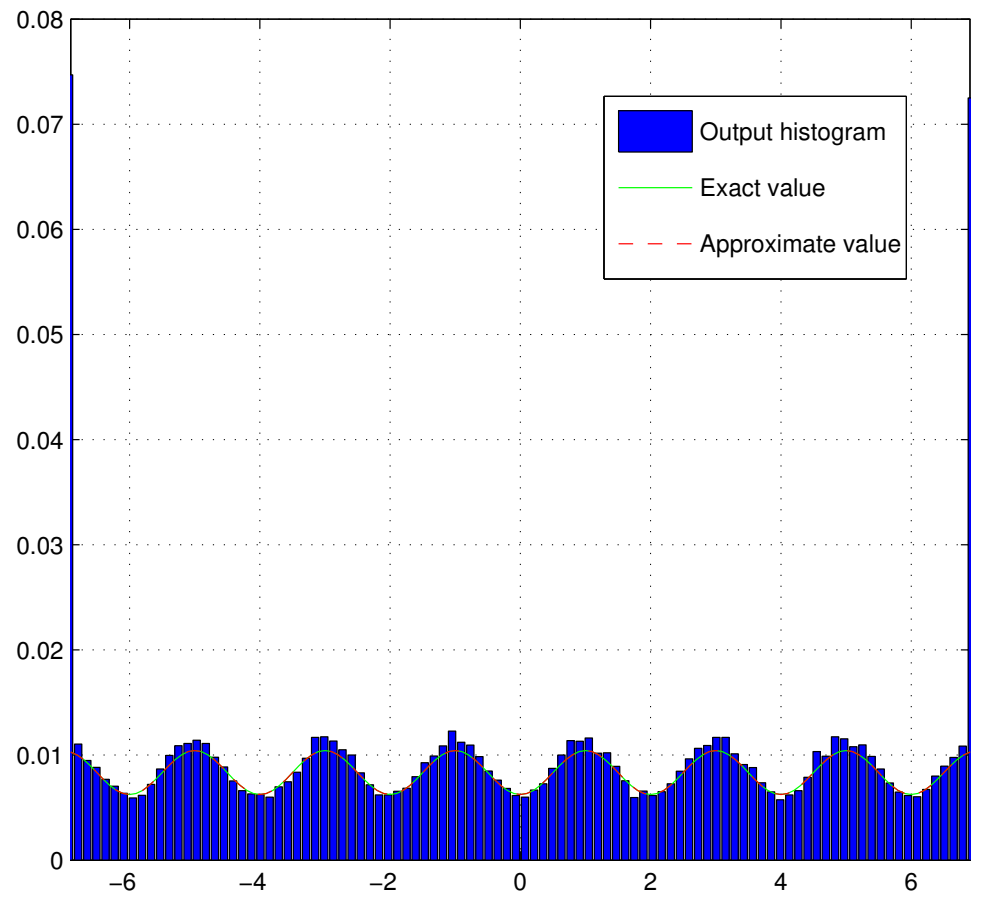

Figure 7: Output statistics for $64 \times 64$ systems with 64 -QAM and $\mathrm{SNR}=20 \mathrm{~dB}$ (low SNR).

MMSE-SIC-LR in terms of BER. We observe that the proposed detector outperforms both MMSE and MMSE-SIC over the whole SNR region and better exploits the receive diversity thanks to joint detection and box constraint effect which reduce the error propagation. At BER $10^{-3}$, the proposed detector outperforms the MMSE by about $7 \mathrm{~dB}$ and the MMSE-SIC by about $1.5 \mathrm{~dB}$. This gain increases with the growth of the SNR values to achieve about $2 \mathrm{~dB}$ at BER $10^{-4}$ compared to the MMSE-SIC. $\left(P_{S I, 2}\right)$ outperforms the MMSE-SIC-LR for medium SNR values (a gain of $0.6 \mathrm{~dB}$ is observed for BER $10^{-3}$ ). The advantage over the MMSE-SIC-LR decreases 


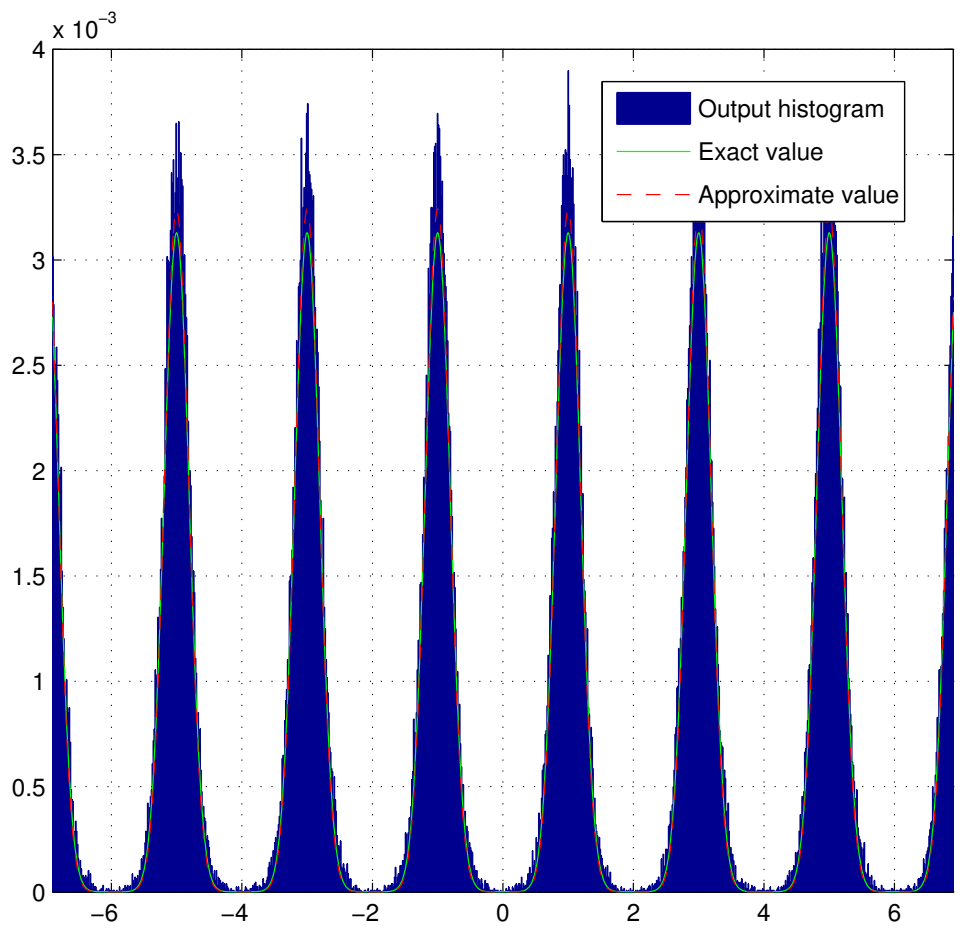

Figure 8: Output statistics for $64 \times 64$ systems with 64 -QAM and SNR=35dB (high SNR).

with increasing SNR and both schemes perform the same for high SNR values. In Fig. 12, we also show that the gain of the proposed scheme $\left(P_{S I, 2}\right)$ over MMSE-based schemes is maintained for higher order modulation. For 16-QAM, the proposed detector outperforms the MMSE-SIC by about $2 \mathrm{~dB}$ for BER $10^{-4}$.

\subsection{Impact of channel estimation inaccuracy}

Channel estimation is a challenging problem in massive MIMO systems as the conventional techniques applicable to MIMO systems cannot be employed owing to an exceptionally large number of unknown channel coefficients. In 


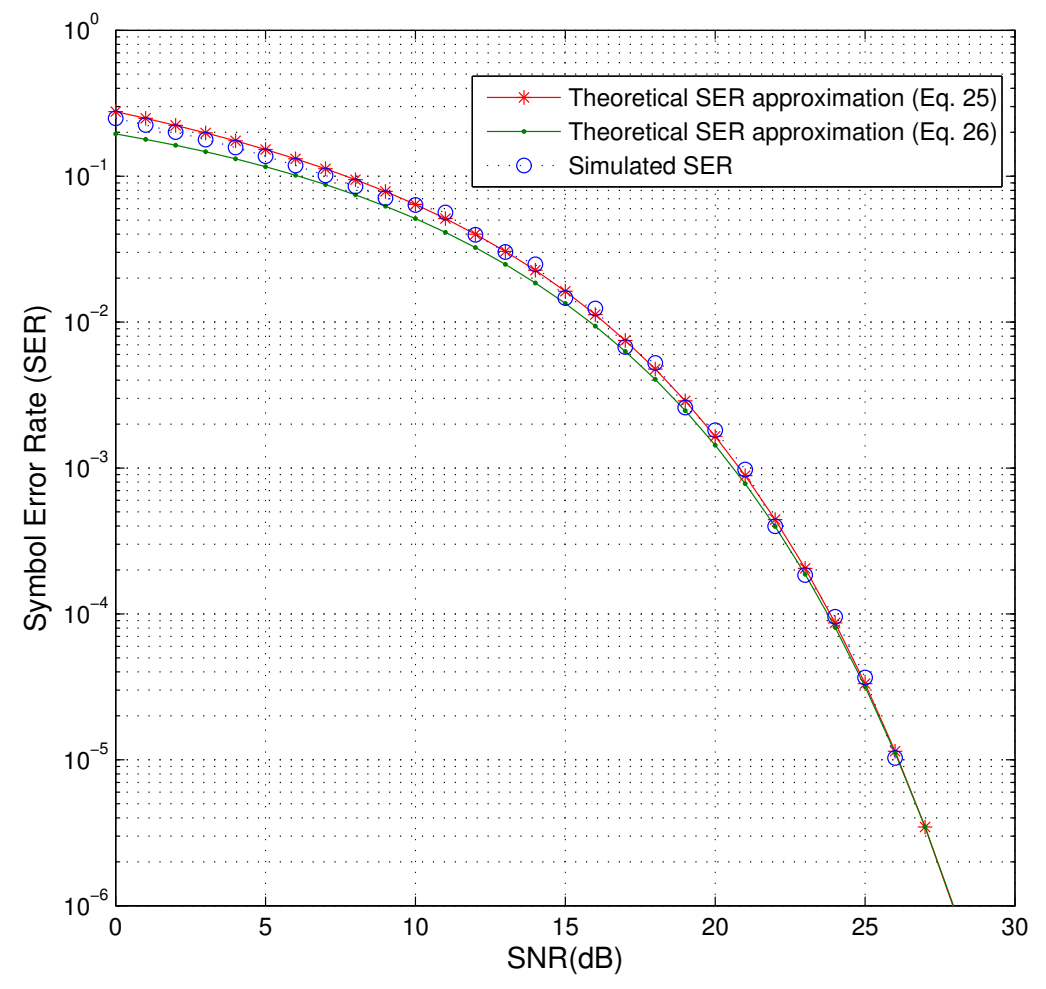

Figure 9: SER performance for $32 \times 32$ systems with 16 -QAM.

order to study the effect of the channel estimation inaccuracy in the determined case, we solve $\left(P_{S I, 2}\right)$ using an estimate of the channel matrix given by $\underline{\widehat{\boldsymbol{H}}}=\underline{\boldsymbol{H}}+\underline{\boldsymbol{H}}_{\epsilon}$ where the estimation error matrix $\underline{\boldsymbol{H}}_{\epsilon}$ is a real Gaussian random matrix such that $\underline{\boldsymbol{H}}_{\epsilon} \sim \mathcal{N}\left(\mathbf{0}, \sigma_{H}^{2} \boldsymbol{I}_{2 N}\right)$ where $\sigma_{H}^{2}$ is the theoretical Mean Square Error (MSE). The simulation setup is summarized in Table 5.

In the determined case, we refer to [38] to compute $\sigma_{H}^{2}$ as a function of the SNR and the system dimensions. The method presented in [38] is an MMSE-based method called O-MMSE. In Fig. 13 we have plotted the performance of the proposed algorithm in the determined case with imperfect 


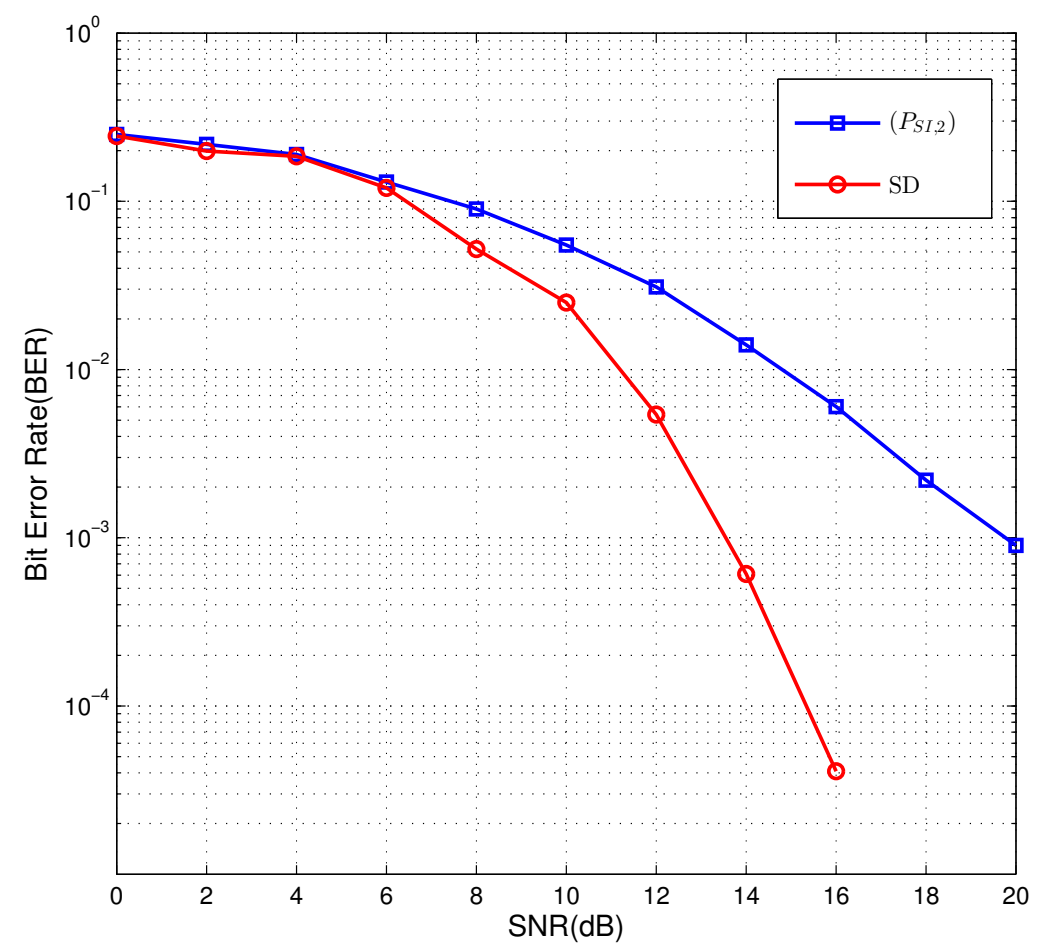

Figure 10: BER performance comparison for $24 \times 18$ systems $\left(\frac{n}{N}=0.75\right)$ with 4-QAM.

CSI estimation compared to the perfect CSI knowledge case. We observe that the $\left(P_{S I, 2}\right)$ algorithm is not very sensitive to channel estimation errors and the loss doesn't exceed $0.4 \mathrm{~dB}$ on the whole SNR range.

Unfortunately, the O-MMSE method can not be applied in the underdetermined case. As a consequence, we propose to evaluate the impact of channel estimation errors by providing both lower and upper bounds of the performance obtained by the best and the worst MSE for the considered SNR range. To that purpose we fix the worst and the best MSE to the MSE obtained by O-MMSE for $\mathrm{SNR}=5 \mathrm{~dB}$ and $\mathrm{SNR}=18 \mathrm{~dB}$ respectively in the case 


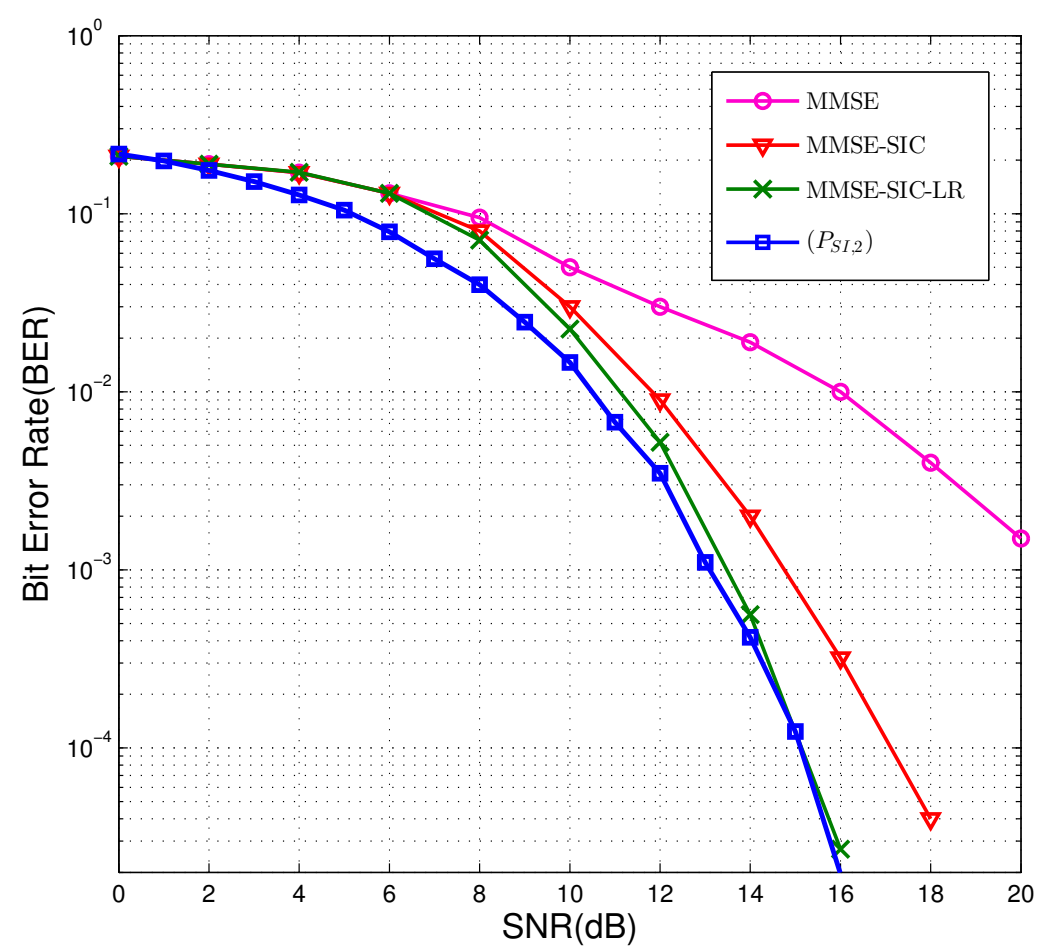

Figure 11: BER performance comparison for $64 \times 64$ systems and 4-QAM.

of $64 \times 64$ systems. The bit error rate is plotted in Fig. 14 where we observe that the imperfect CSI introduces a slight performance degradation.

We can conclude that the proposed detection is quite robust to CSI inaccuracy.

\section{Conclusions}

This paper focused on finite-alphabet source signal recovery in large-scale MIMO systems. We first proposed a simplicity-based $\ell_{1}$-minimization combined with box constraints to solve the noise-free case. For the proposed cri- 


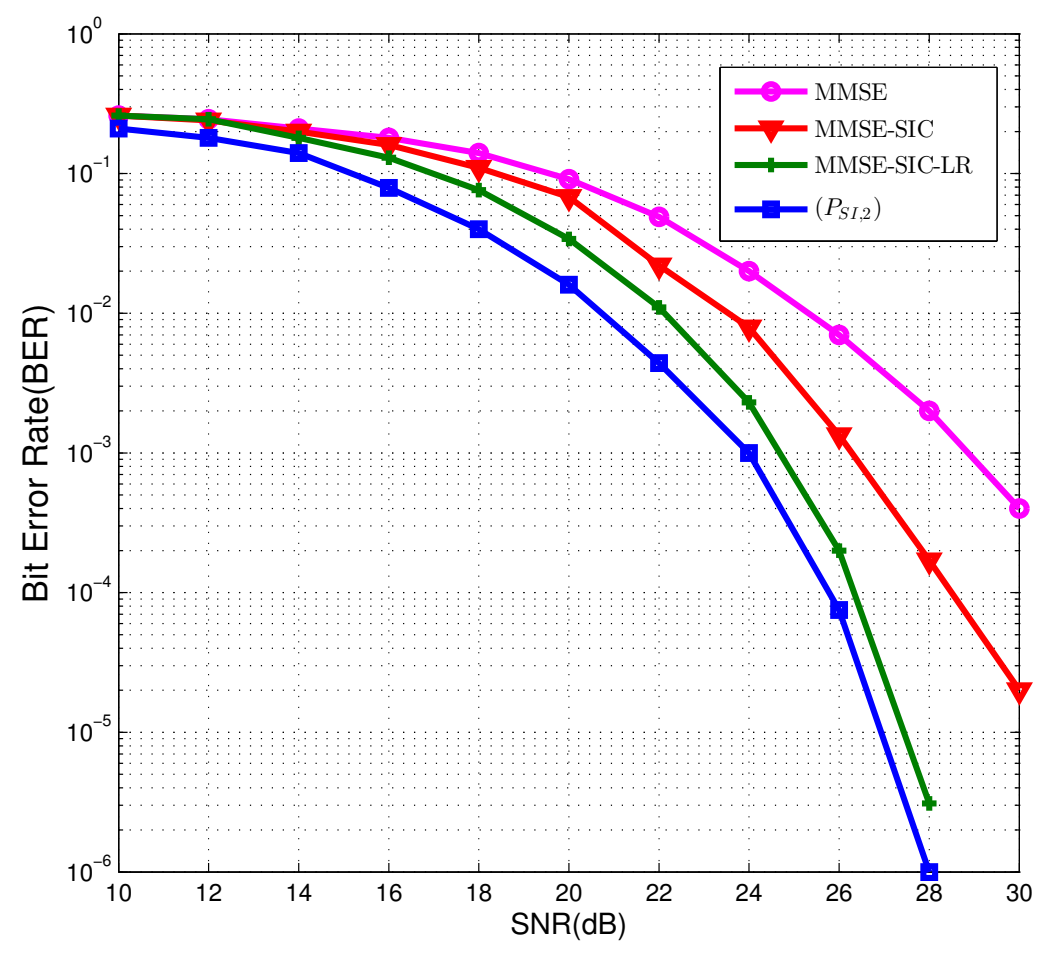

Figure 12: BER performance comparison in $64 \times 64$ systems and 16-QAM.

terion, we investigated the necessary condition of uniqueness and existence of a solution which is given by $\frac{n}{N}>\frac{p-1}{p}$ (see Statement $(\boldsymbol{i i})$ of Theorem 3.1). This condition covers the determined case and partially the underdetermined case. Compared to previous existing sparsity-based techniques, we obtained a sufficient computation cost reduction with recovery success rate preservation. Simulation results corroborated the theoretical analysis. By exploiting the necessary condition of successful recovery on the problem parameters, we studied performance of the proposed criterion in a more practical case by considering the noisy massive MIMO system with both perfect and imper- 


\begin{tabular}{|l|l|}
\hline Parameters & Value \\
\hline \hline Mixing matrix $\boldsymbol{H}$ & $H_{i j} \sim \mathcal{C N}(0,1)$ i.i.d for $\forall i, j$ \\
\hline Mixing matrix knowledge & MMSE estimation or model error \\
\hline QAM modulation order $M$ & $M=4$ \\
\hline Number of sources $N$ & $N=64$ \\
\hline Number of observations $n$ & $n \in\{62,64\}$ \\
\hline Matlab toolbox & CVX toolbox \\
\hline
\end{tabular}

Table 5: Simulation setup for Section 4.4.

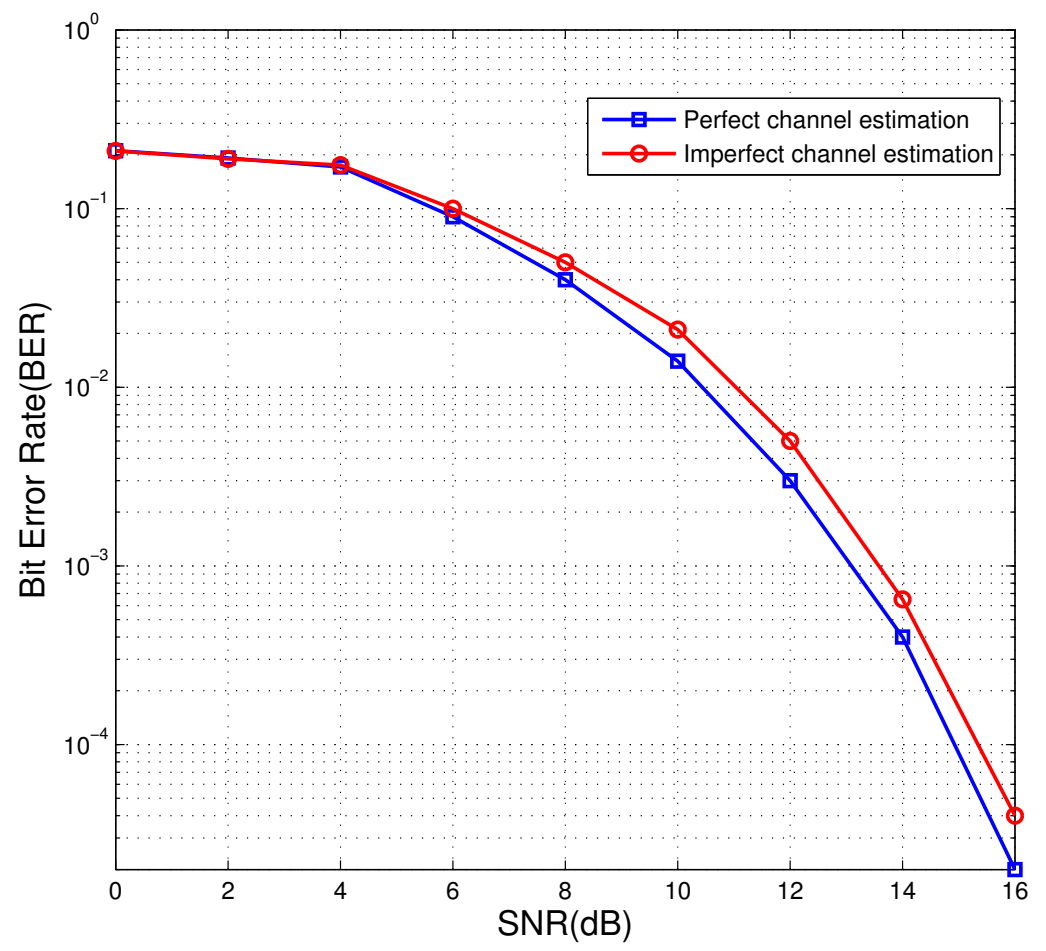

Figure 13: BER performance comparison in $64 \times 64$ systems and 4-QAM. 


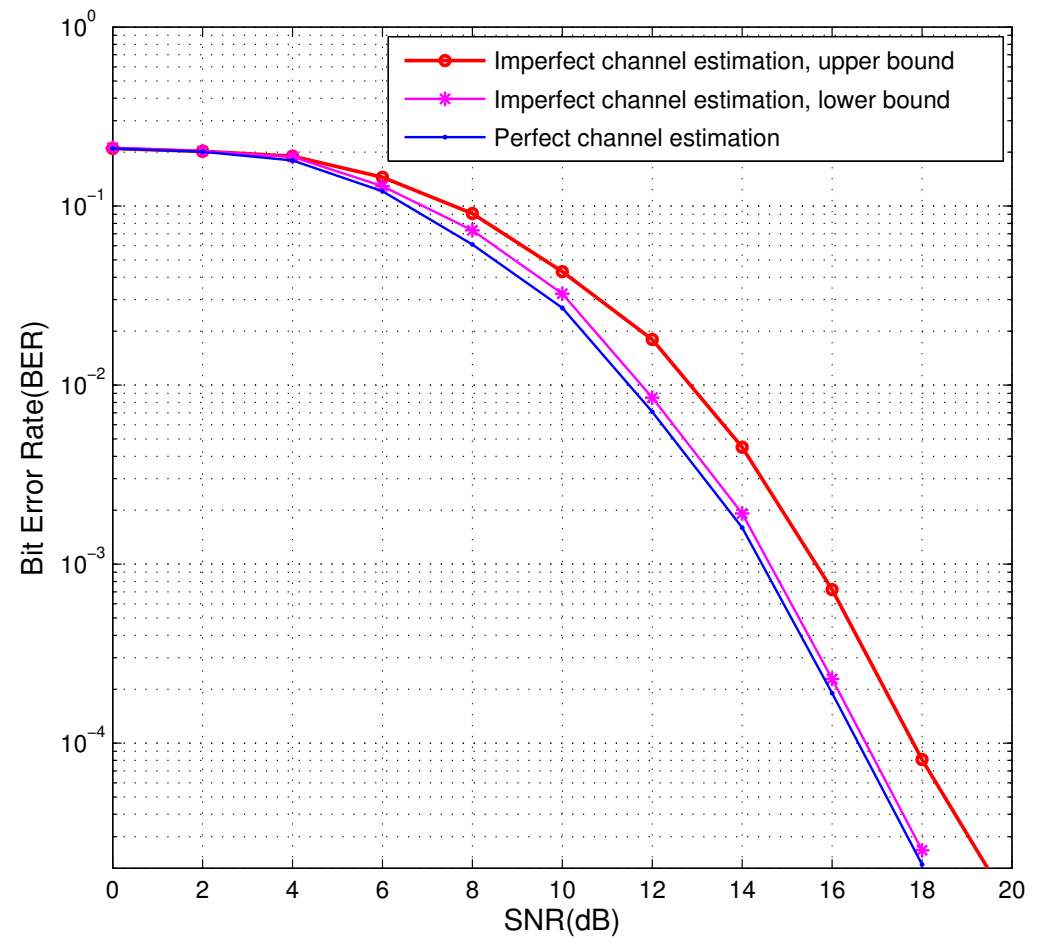

Figure 14: BER performance comparison in $64 \times 62$ systems and 4-QAM.

fect CSI knowledge. The low-complexity resulting algorithm is well-adapted to such applications and its computation cost doesn't depend on the constellation size. The theoretical distribution of the detector output was then validated through simulations. In the future we expect to use the analytical results to introduce the proposed algorithm to define an iterative shadow area-based detection to further improve the performance or to define a turbolike iterative receiver to take into account an outer forward error corrcetion code. 


\section{Appendix A. Generic random matrix $\underline{H}$}

We assume that the components of $\boldsymbol{H}$ are independent, complex circularlysymmetric Gaussian random variables with zero mean and unit variance. We aim to prove that $\underline{\boldsymbol{H}}$ is a generic random matrix. The assumption on the distribution of the components of $\boldsymbol{H}$ ensures that the columns of $\underline{\boldsymbol{H}}$ are symmetrically distributed about the origin. We thus have to prove that $\underline{\boldsymbol{H}}$

is completely general with probability 1 , that is to say whatever $\ell$, any $\ell \times \ell$ submatrix of $\underline{\boldsymbol{H}}$ has full-rank. This result is given by the following Theorem A.1.

Theorem A.1 : Given a complex-valued matrix $\boldsymbol{H}$ and its real-valued transform $\underline{\boldsymbol{H}}$, if $\boldsymbol{H}$ is a generic random matrix with independent circularlysymmetric Gaussian-distributed components, then $\underline{\boldsymbol{H}}$ is completely general with probability 1 .

Let us prove the theorem by induction. Let $\boldsymbol{H}$ be a complex-valued matrix with independent circularly-symmetric Gaussian-distributed components. Let us define the property $\mathcal{P}_{\ell}$ by "any $\ell \times \ell$ submatrix of $\underline{\boldsymbol{H}}$ has full-rank with probability 1 ". Let $\ell=1$. Then any $1 \times 1$ submatrix of $\underline{\boldsymbol{H}}$ is a real-valued Gaussian variable, that is to say a continuous random variable and the probability that it equals zero is null. $\mathcal{P}_{1}$ is true. Let us suppose that $\mathcal{P}_{\ell}$ is true and let us prove that $\mathcal{P}_{\ell+1}$ is also true. Let $S$ an $(\ell+1) \times(\ell+1)$ submatrix of $\underline{\boldsymbol{H}}$. Then as $\mathcal{P}_{\ell}$ is true, all minors of $\boldsymbol{S}$ have non-zero determinant with probability 1. Let us compute the determinant of $\boldsymbol{S}$ according to a given row (or column). It corresponds to the linear combination of minors, where due to the independence of the components of $\underline{\boldsymbol{H}}$ (the components of $\boldsymbol{H}$ are circularly-symmetric Gaussian and independent) the weights and the minors 
form a family of random variables that are mutually independent, continuously distributed and different from zero with probability equal to one. Thus the determinant of $\boldsymbol{S}$ is a continuous random variable different from zero with probability equal to one.

\section{Appendix B. Proof of Proposition 4.1}

The number of non binding constraints of $\left(P_{S I, 2}\right)$ can be seen as the sum of the inactive constraint number and the non binding active constraint number. The probability that a constraint is inactive is denoted by $p_{\text {in }}$ and corresponds to the probability that $\underline{x}_{i} \notin\left\{\alpha_{1}, \alpha_{p}\right\}$, that is to say $p_{i n}=\frac{p-2}{p}$. The probability that a constraint is non binding and active is denoted by $p_{n b a}$ and corresponds to the probability that either $\underline{x}_{i}=\alpha_{1}$ and $\left\{\underline{\boldsymbol{H}}^{T}(\underline{\boldsymbol{H} \hat{\boldsymbol{x}}}-\underline{\boldsymbol{y}})\right\}_{i}<0$, or $\underline{x}_{i}=\alpha_{p}$ and $\left.\left\{\underline{\boldsymbol{H}}^{T}(\underline{\boldsymbol{H} \hat{\boldsymbol{x}}}-\underline{\boldsymbol{y}})\right\}_{i}>0\right\}$. As a constraint cannot be active and inactive, the probability that a constraint is non binding equals $p_{n b}=p_{i n}+p_{n b a}$.

It remains to find the value of $p_{n b a}=\operatorname{Pr}(i \in \Omega \backslash \Lambda)$. To that purpose we focus on the sign of $\left\{\underline{\boldsymbol{H}}^{T}(\underline{\boldsymbol{H} \hat{\boldsymbol{x}}}-\underline{\boldsymbol{y}})\right\}_{i}=\left\{\underline{\boldsymbol{H}}^{T}(\underline{\boldsymbol{H}}(\underline{\hat{\boldsymbol{x}}}-\underline{\boldsymbol{x}})-\boldsymbol{\zeta})\right\}_{i}, i \in \Lambda$. As the elements in the set $\mathcal{F}=\left\{\alpha_{1}, \alpha_{2}, . ., \alpha_{p}\right\}$ are equiprobable and the real-valued matrix channel $\underline{\boldsymbol{H}}$ as well as the noise are Gaussian, we can affirm that the estimated vector $\underline{\hat{\boldsymbol{x}}}$ is a symmetrically erroneous version of the original vector $\underline{\boldsymbol{x}}$. Then the sign of $\{(\underline{\hat{\boldsymbol{x}}}-\underline{\boldsymbol{x}})\}_{i}, i \in \Lambda$ takes on equiprobable values. Consequently exploiting the same hypothesis for the channel matrix and the noise we deduce that the sign of $\left\{\underline{\boldsymbol{H}}^{T}(\underline{\boldsymbol{H} \hat{\boldsymbol{x}}}-\underline{\boldsymbol{y}})\right\}_{i}$ can be negative or positive 
with probability $1 / 2$. Then $p_{n b a}$ can be decomposed as:

$$
\begin{aligned}
p_{n b a} & =\operatorname{Pr}\left(\underline{x}_{i}=\alpha_{1}\right) \operatorname{Pr}\left(\left\{\tilde{\boldsymbol{H}}^{T}(\underline{\boldsymbol{H} \hat{\boldsymbol{x}}}-\underline{\boldsymbol{y}})\right\}_{i} \leq 0 \mid \underline{x}_{i}=\alpha_{1}\right) \\
& +\operatorname{Pr}\left(\underline{x}_{i}=\alpha_{p}\right) \operatorname{Pr}\left(\left\{\underline{\boldsymbol{H}}^{T}(\underline{\boldsymbol{H} \hat{\boldsymbol{x}}}-\underline{\boldsymbol{y}})\right\}_{i} \geq 0 \mid \underline{x}_{i}=\alpha_{p}\right) \\
& =\frac{1}{p} \times \frac{1}{2}+\frac{1}{p} \times \frac{1}{2}=\frac{1}{p} .
\end{aligned}
$$

Consequently $p_{n b}=p_{i n}+p_{n b a}=\frac{p-2}{p}+\frac{1}{p}=\frac{p-1}{p}$, Hence $\operatorname{card}(\bar{\Lambda}) \sim \mathcal{B}\left(2 N, \frac{p-1}{p}\right)$.

\section{Appendix C. Symbol error probability upper-bound}

Proof of Theorem 4.3. Let us denote by $\underline{\tilde{x}}_{k}$ the hard decision taken on $\underline{x}_{k}$ from the detection output $\underline{\hat{x}}_{k}$. Then the symbol error probability is defined by

$$
P_{s}=\operatorname{Pr}\left(\underline{x}_{k} \neq \underline{\tilde{x}}_{k}\right) .
$$

Considering the assumptions (alphabet and equiprobability), $P_{s}$ reads

$$
P_{s}=\frac{1}{p} \sum_{i=1}^{p} \sum_{\substack{q=1 \\ i \neq q}}^{p} \operatorname{Pr}\left(\underline{\tilde{x}}_{k}=\alpha_{q} \mid \underline{x}_{k}=\alpha_{i}\right) .
$$

This probability can be computed by considering a maximum-likelihood decision rule applied on $\underline{\hat{x}}_{k}$ :

$$
\begin{aligned}
P_{s} & =\frac{1}{p} \sum_{i=1}^{p} \sum_{\substack{q=1 \\
i \neq q}}^{p} \operatorname{Pr}\left(\bigcap_{\substack{j=1 \\
j \neq q}}^{p}\left(\left(\underline{\hat{x}}_{k}-\alpha_{q}\right)^{2} \leq\left(\underline{\hat{x}}_{k}-\alpha_{j}\right)^{2}\right) \mid \underline{x}_{k}=\alpha_{i}\right) \\
& \leq \frac{1}{p} \sum_{i=1}^{p} \sum_{\substack{q=1 \\
i \neq q}}^{p} \operatorname{Pr}\left(\left(\underline{\hat{x}}_{k}-\alpha_{q}\right)^{2} \leq\left(\underline{\hat{x}}_{k}-\alpha_{i}\right)^{2} \mid \underline{x}_{k}=\alpha_{i}\right) \\
& \leq \frac{1}{p} \sum_{i=1}^{p} \sum_{\substack{q=1 \\
i \neq q}}^{p} \operatorname{Pr}\left(\underline{\hat{x}}_{k} \leq \frac{\alpha_{i}+\alpha_{q}}{2} \mid \underline{x}_{k}=\alpha_{i}\right) .
\end{aligned}
$$


Using Theorem 4.2, we can write

$$
\begin{aligned}
& \operatorname{Pr}\left(\underline{\hat{x}}_{k} \leq \frac{\alpha_{i}+\alpha_{q}}{2} \mid \underline{x}_{k}=\alpha_{i}\right)=\frac{1}{2} \operatorname{erfc}\left(\frac{\alpha_{i}-\alpha_{1}}{\sqrt{2} \sigma_{\underline{\hat{x}}}}\right) \\
& +\frac{1}{2} \operatorname{erfc}\left(\frac{\alpha_{p}-\alpha_{i}}{\sqrt{2} \sigma_{\underline{\hat{x}}}}\right)+\int_{\alpha_{1}}^{\frac{\alpha_{i}+\alpha_{q}}{2}} \frac{1}{\sqrt{2 \pi} \sigma_{\underline{\hat{x}}}} \exp \left(-\frac{\left(x-\alpha_{i}\right)^{2}}{2 \sigma_{\underline{\hat{x}}}^{2}}\right) d x .
\end{aligned}
$$

After computation, we get

$$
\operatorname{Pr}\left(\underline{\hat{x}}_{k} \leq \frac{\alpha_{i}+\alpha_{q}}{2} \mid \underline{x}_{k}=\alpha_{i}\right)=\frac{1}{2} \operatorname{erfc}\left(\frac{\alpha_{p}-\alpha_{i}}{\sqrt{2} \sigma_{\underline{\hat{x}}}}\right)+\frac{1}{2} \operatorname{erfc}\left(\frac{\alpha_{i}-\alpha_{q}}{2 \sqrt{2} \sigma_{\underline{\hat{x}}}}\right) .
$$

Therefore $P_{s}$ can be upper-bounded by

$$
P_{s} \leq \frac{1}{2 p} \sum_{i=1}^{p} \sum_{\substack{q=1 \\ i \neq q}}^{p} \operatorname{erfc}\left(\frac{\alpha_{i}-\alpha_{q}}{2 \sqrt{2} \sigma_{\underline{\hat{x}}}}\right)+\frac{p-1}{2 p} \sum_{i=1}^{p} \operatorname{erfc}\left(\frac{\alpha_{p}-\alpha_{i}}{\sqrt{2} \sigma_{\underline{\hat{x}}}}\right) .
$$

At high SNR, due to the decreasing rate of erfc, the terms depending on differences between adjacent symbols are predominant and the following approximation is asymptotically tight

$$
P_{s} \approx \frac{p-1}{p} \operatorname{erfc}\left(\frac{\alpha_{2}-\alpha_{1}}{2 \sqrt{2} \sigma_{\underline{\hat{x}}}}\right) \text {. }
$$

\section{References}

[1] N. Mourad, J. P. Reilly, T. Kirubarajan, Majorization minimization for blind source separation of sparse sources, Signal Processing 131 (2017) 120 - 133. doi:10.1016/j.sigpro.2016.08.015. 
[2] C. Chenot, J. Bobin, Blind separation of sparse sources in the presence of outliers, Signal Processing 138 (2017) 233 - 243. doi:10.1016/j.sigpro.2017.03.024.

[3] S. Rambhatla, J. Haupt, Semi-blind source separation via sparse representations and online dictionary learning, in: Asilomar Conference on Signals, Systems and Computers, 2013, pp. 1687-1691. doi:10.1109/ACSSC.2013.6810587.

[4] D. L. Donoho, Compressed sensing, IEEE Transactions on Information Theory 52 (4) (2006) 1289-1306. doi:10.1109/TIT.2006.871582.

[5] N. B. Karahanoglu, H. Erdogan, Compressed sensing signal recovery via forward-backward pursuit, Digital Signal Processing 23 (5) (2013) 1539 - 1548. doi:10.1016/j.dsp.2013.05.007.

[6] T. Liu, T. Qiu, R. Dai, J. Li, L. Chang, R. Li, Nonlinear regression $\mathrm{A}^{*} \mathrm{OMP}$ for compressive sensing signal reconstruction, Digital Signal Processing 69 (Supplement C) (2017) 11 - 21. doi:10.1016/j.dsp.2017.06.004.

[7] E. J. Candès, J. K. Romberg, T. Tao, Stable signal recovery from incomplete and inaccurate measurements, Communications on Pure and Applied Mathematics 59 (8) (2006) 1207-1223. doi:10.1002/cpa.20124.

[8] K. Liu, Z. Xu, X. Xi, S. Wang, Sparse signal reconstruction via concave continuous piecewise linear programming, Digital Signal Processing 54 (Supplement C) (2016) 12 - 26. doi:10.1016/j.dsp.2016.03.010. 
[9] H. Cui, H. Duan, Sparse bayesian learning using correlated hyperparameters for recovery of block sparse signals, Digital Signal Processing 68 (2017) 24 - 30. doi:10.1016/j.dsp.2017.05.003.

[10] A. Aïssa-El-Bey, D. Pastor, S. Aziz Sbaï, Y. Fadlallah, Sparsity-based recovery of finite alphabet solutions to underdetermined linear systems, IEEE Transactions on Information Theory 61 (4) (2015) 2008-2018. doi:10.1109/TIT.2015.2399914.

[11] O. L. Mangasarian, B. Recht, Probability of unique integer solution to a system of linear equations, European Journal of Operational Research 214 (1) (2011) 27-30. doi:10.1016/j.ejor.2011.04.010.

[12] D. L. Donoho, J. Tanner, Counting faces of randomly-projected polytopes when the projection radically lowers dimension, Journal of the American Mathematical Society 22 (1) (2009) 1-53.

[13] Y. Fadlallah, A. Aïssa-El-Bey, K. Amis, D. Pastor, R. Pyndiah, New iterative detector of MIMO transmission using sparse decomposition, IEEE Transactions on Vehicular Technology 64 (8) (2015) 3458-3464. doi:10.1109/TVT.2014.2360687.

[14] Y. Fadlallah, A. Aïssa-El-Bey, K. Amis, D. Pastor, R. Pyndiah, New decoding strategy for underdetermined MIMO transmission using sparse decomposition, in: Proceedings of the 21st European Signal Processing Conference (EUSIPCO), 2013, pp. 1-5.

[15] B. Panzner, W. Zirwas, S. Dierks, M. Lauridsen, P. Mogensen, K. Pajukoski, D. Miao, Deployment and implementation strategies for mas- 
sive MIMO in 5G, in: IEEE Globecom Workshops, 2014, pp. 346-351. doi:10.1109/GLOCOMW.2014.7063455.

[16] B. Hassibi, H. Vikalo, On the sphere-decoding algorithm I. expected complexity, IEEE Transactions on Signal Processing 53 (8) (2005) 28062818. doi:10.1109/TSP.2005.850352.

[17] L. B. White, MMSE precoder design for diversity systems with temporal correlation, Digital Signal Processing 16 (5) (2006) 607 - 618, special Issue on DASP 2005. doi:10.1016/j.dsp.2005.02.006.

[18] X. He, Q. Guo, J. Tong, J. Xi, Y. Yu, Low-complexity approximate iterative LMMSE detection for large-scale MIMO systems, Digital Signal Processing 60 (Supplement C) (2017) 134 - 139. doi:10.1016/j.dsp.2016.09.004.

[19] A. H. Mehana, A. Nosratinia, Performance of MIMO single-carrier frequency domain zero-forcing equalizer, in: IEEE International Symposium on Information Theory Proceedings (ISIT), 2012, pp. 3023-3027. doi:10.1109/ISIT.2012.6284116.

[20] B. Hassibi, An efficient square-root algorithm for BLAST, in: IEEE International Conference on Acoustics, Speech, and Signal Processing (ICASSP), Vol. 2, 2000, pp. 737-740. doi:10.1109/ICASSP.2000.859065.

[21] D. Wubben, R. Bohnke, V. Kuhn, K. D. Kammeyer, MMSE-based lattice-reduction for near-ML detection of MIMO systems, in: ITG Workshop on Smart Antennas (IEEE Cat. No.04EX802), 2004, pp. 106113. 
[22] J. G. Wendel, A problem in geometric probability, Mathematica Scandinavica 11 (1962) 109-112. doi:10.7146/math.scand.a-10655.

[23] S. S. Chen, D. L. Donoho, M. A. Saunders, Atomic decomposition by basis pursuit, SIAM Journal on Scientific Computing 20 (1) (1998) 3361. doi:10.1137/S1064827596304010.

[24] Z. Hajji, K. Amis Cavalec, A. Aïssa-El-Bey, F. Abdelkefi, Lowcomplexity half-sparse decomposition-based detection for massive MIMO transmission, in: 5th International Conference on Communications and Networking (ComNet), 2015, pp. 1-6. doi:10.1109/COMNET.2015.7566636.

[25] X. Fan, J. Song, D. P. Palomar, O. C. Au, Universal binary semidefinite relaxation for $\mathrm{ml}$ signal detection, IEEE Transactions on Communications 61 (11) (2013) 4565-4576. doi:10.1109/TCOMM.2013.092013.120988.

[26] T. Cui, C. Tellambura, An efficient generalized sphere decoder for rankdeficient MIMO systems, IEEE Communications Letters 9 (5) (2005) 423-425. doi:10.1109/LCOMM.2005.1431159.

[27] H. Karloff, The Simplex Algorithm, Birkhäuser Boston, Boston, MA, 1991, Ch. 2, pp. 23-47. doi:10.1007/978-0-8176-4844-2_2.

[28] Y. Nesterov, A. Nemirovskii, Interior-Point Polynomial Algorithms in Convex Programming, Path-Following Interior-Point Methods, Society for Industrial and Applied Mathematics, 1994, Ch. 3, pp. 57-99. doi:10.1137/1.9781611970791.ch3. 
[29] D. L. Donoho, J. Tanner, Counting the faces of randomly-projected hypercubes and orthants, with applications, Discrete \& Computational Geometry 43 (3) (2010) 522-541. doi:10.1007/s00454-009-9221-z.

[30] M. Grant, S. Boyd, CVX: Matlab software for disciplined convex programming, version 2.1, http://cvxr.com/cvx (March 2014).

[31] M. Grant, S. Boyd, Graph implementations for nonsmooth convex programs, in: V. Blondel, S. Boyd, H. Kimura (Eds.), Recent Advances in Learning and Control, Lecture Notes in Control and Information Sciences, Springer-Verlag Limited, 2008, pp. 95-110, http: //stanford.edu/ boyd/graph_dcp.html.

[32] E. D. Andersen, C. Roos, T. Terlaky, On implementing a primal-dual interior-point method for conic quadratic optimization, Mathematical Programming 95 (2) (2003) 249-277. doi:10.1007/s10107-002-0349-3.

[33] P. B. Stark, R. L. Parker, Bounded-variable least-squares: an algorithm and applications, Computational Statistics 10 (2) (1995) 129-141.

[34] K. V. Mardia, J. T. Kent, J. M. Bibby, Multivariate analysis, Academic press, 1980.

[35] K. Cho, D. Yoon, On the general ber expression of one- and twodimensional amplitude modulations, IEEE Transactions on Communications 50 (7) (2002) 1074-1080. doi:10.1109/TCOMM.2002.800818.

[36] Y. H. Gan, W. H. Mow, Complex lattice reduction algorithms for low-complexity MIMO detection, in: IEEE Global Telecommu- 
nications Conference (GLOBECOM), Vol. 5, 2005, pp. 2953-2957. doi:10.1109/GLOCOM.2005.1578299.

[37] I. Gurobi Optimization, Gurobi optimizer reference manual (2015). URL http://www.gurobi.com

[38] A. Zaib, M. Masood, A. Ali, W. Xu, T. Y. Al-Naffouri, Distributed channel estimation and pilot contamination analysis for massive MIMOOFDM systems, IEEE Transactions on Communications 64 (11) (2016) 4607-4621. doi:10.1109/TCOMM.2016.2593924.

\section{Thanks}

The authors would like to thank the Brittany Region and PRACOM for their financial support.

\section{Authors' Biographies}

Zahran HAJJI was born in Djerba, Tunisia in December 1992. He received the Dr. Eng. degree in Telecommunications Engineering in September 2015 from Higher School of Communication of Tunis (SupCom). Since November 2015, he has been pursuing the Ph.D. degree at the the Signal and Communication department of Telecom Bretagne, Brest, France. His main research interests include wireless communications, massive MIMO technologies, iterative decoding and detection and compressed sensing.

Abdeldjalil Aïssa-El-Bey received the State Engineering degree from Ecole Nationale Polytechnique (ENP), Algiers, Algeria, in 2003, the M.S. 
degree in signal processing from Supelec and Paris XI University, Orsay, France, in 2004, and the Ph.D. degree in signal and image processing from ENST Paris, France, in 2007. In 2007, he joined the Signal and Communications department of IMT Atlantique (Telecom Bretagne), Brest, France as an Associate Professor, and then Professor, since 2015. He was a Visiting Researcher at Fujitsu Laboratories, Japan and the department of Electrical and Electronic Engineering of The University of Melbourne, Australia in 2010 and 2015, respectively. His research interests are blind source separation, blind system identification and equalization, compressed sensing, sparse signal processing, statistical signal processing, wireless communications, and adaptive filtering.

Karine Amis received her Dipl.-Ing. degree from the Ecole Nationale Superieure des Telecommunications de Bretagne (ENST Bretagne), France in 1998, her Ph.D. degree from the University of Rennes 1, France in 2001 and the accreditation to supervise research ( $\mathrm{HdR}$ ) from the University of western Brittany (UBO) in 2014. Since 2001, she is an associate professor at IMT Atlantique (ex-Telecom Bretagne), France. Her current research interests focus on communication and coding theory with application to MIMO systems, interference channels, cooperative communications and sporadic communications. 